Homology, Homotopy and Applications, vol.15(1), 2013, pp.191-222

\title{
CONTINUOUS HOMOTOPY FIXED POINTS FOR LUBIN-TATE SPECTRA
}

\author{
GEREON QUICK
}

(communicated by Daniel Dugger)

\begin{abstract}
We provide a new and conceptually simplified construction of continuous homotopy fixed point spectra for Lubin-Tate spectra under the action of the extended Morava stabilizer group. Moreover, our new construction of a homotopy fixed point spectral sequence converging to the homotopy groups of the homotopy fixed points of Lubin-Tate spectra is isomorphic to an Adams spectral sequence converging to the homotopy groups of the spectra constructed by Devinatz and Hopkins. The new idea is built on the theory of profinite spectra with a continuous action by a profinite group.
\end{abstract}

\section{Introduction}

For the action of a discrete group $G$ on a spectrum $X$ there are well-known constructions for the homotopy fixed point spectrum $X^{h G}$ and for the homotopy fixed point spectral sequence. For a spectrum $X$, the spectrum $X^{h G}$ is given by the $G$ fixed points of the function spectrum $F\left(E G_{+}, R X\right)$, where $E G$ is a contractible free $G$-space and $R X$ denotes a fibrant replacement of $X$. For each spectrum $Z$, the spectral sequence

$$
H^{*}\left(G ; X^{*} Z\right) \Rightarrow\left[Z, X^{h G}\right]^{*}
$$

is induced by the filtration by the finite subskeleta of $E G$. But in some cases of interest, the group $G$ and the spectrum $X$ carry additional structures that one would like to take care of. For example, this is the case for the most important group action in the chromatic approach to stable homotopy theory, the action of the extended Morava stabilizer group $G_{n}$ on the $p$-local Landweber exact spectrum $E_{n}$. Let us briefly describe this well-known example.

Let $p$ be a fixed prime, $n \geqslant 1$ an integer and $\mathbb{F}_{p^{n}}$ the field with $p^{n}$ elements. Let $S_{n}$ be the $n$th Morava stabilizer group, i.e., the automorphism group of the height $n$ Honda formal group law $\Gamma_{n}$ over $\mathbb{F}_{p^{n}}$. We denote by $\operatorname{Gal}\left(\mathbb{F}_{p^{n}} / \mathbb{F}_{p}\right)$ the Galois group of

The author was supported by Research Fellowship QU 317/1 of the German Research Foundation (DFG).

Received March 13, 2011, revised October 21, 2012; published on April 28, 2013.

2000 Mathematics Subject Classification: 55P43, 55Q52, 55Q91, 55T15.

Key words and phrases: homotopy fixed point, Lubin-Tate spectrum, Morava stabilizer group, Adams spectral sequence.

Article available at http://intlpress.com/HHA/v15/n1/a10 and doi:10.4310/HHA.2013.v15.n1.a10

Copyright (C) 2013, International Press. Permission to copy for private use granted. 
$\mathbb{F}_{p^{n}}$ over $\mathbb{F}_{p}$ and let $G_{n}=S_{n} \rtimes \operatorname{Gal}\left(\mathbb{F}_{p^{n}} / \mathbb{F}_{p}\right)$ be the semi-direct product. By the work of Lubin and Tate [18], there is a universal ring of deformations

$$
E\left(\mathbb{F}_{p^{n}}, \Gamma_{n}\right)=W\left(\mathbb{F}_{p^{n}}\right)\left[\left[u_{1}, \ldots, u_{n-1}\right]\right]
$$

of $\left(\mathbb{F}_{p^{n}}, \Gamma_{n}\right)$, where $W\left(\mathbb{F}_{p^{n}}\right)$ denotes the ring of Witt vectors of $\mathbb{F}_{p^{n}}$. The $M U_{*}$-module $E\left(\mathbb{F}_{p^{n}}, \Gamma_{n}\right)\left[u, u^{-1}\right]$ induces via the Landweber exact functor theorem a homology theory and hence a spectrum, denoted by $E_{n}$ and called the Lubin-Tate spectrum, with $E_{n *}=E\left(\mathbb{F}_{p^{n}}, \Gamma_{n}\right)\left[u, u^{-1}\right],|u|=-2$. The profinite group $G_{n}$ acts on the ring $E_{n *}$ (cf. [7]). By Brown representability, this induces an action of $G_{n}$ by maps of rings in the stable homotopy category. Furthermore, Goerss, Hopkins and Miller have shown the crucial fact that there is even a $G_{n}$-action on the spectrum-level on $E_{n}$ that induces the action in the stable category (see [11] and $[\mathbf{2 6}]$ ).

Now $S_{n}, \operatorname{Gal}\left(\mathbb{F}_{p^{n}} / \mathbb{F}_{p}\right)$ and hence also $G_{n}$ are profinite groups. Moreover each homotopy group $\pi_{t} E_{n}$ has the structure of a continuous profinite $G_{n}$-module. The continuity of the action of $G_{n}$ on each $\pi_{t} E_{n}$ is an important property for stable homotopy theory. For by Morava's change of rings theorem, the $K(n)_{*}$-local $E_{n}$-Adams spectral sequence for the sphere spectrum $S^{0}$ has the form

$$
H^{*}\left(G_{n} ; E_{n *}\right) \Rightarrow \pi_{*} L_{K(n)} S^{0}
$$

where the $E_{2}$-term is continuous cohomology of $G_{n}$ with profinite coefficients $E_{n *}$. Here $K(n)$ denotes the $n$th Morava $K$-theory and $L_{K(n)}$ denotes $K(n)_{*}$-localization (cf. [21] and [5]). Hence $L_{K(n)} S^{0}$ looks like a continuous $G_{n}$-homotopy fixed point spectrum of $E_{n}$ and one would like to interpret the above spectral sequence as a continuous homotopy fixed point spectral sequence of the $G_{n}$-action.

But the classical construction of homotopy fixed points and its spectral sequence (1) do not reflect the topology on $G_{n}$. The function spectrum $F\left(E G_{+}, E_{n}\right)$ should consist of continuous maps in some sense and the $E_{2}$-term of the spectral sequence (1) should be continuous cohomology of $G$. Hence it is a fundamental question in stable homotopy theory to understand in which way $E_{n}$ can be viewed as an object with a continuous action under $G_{n}$.

Devinatz and Hopkins [8] have circumvented this problem and given an ad hoc argument for the construction of a spectrum that has the expected homotopy type of the continuous homotopy fixed points of $E_{n}$. They proceeded in two steps by first constructing a spectrum, here denoted by $E_{n}^{d h U}$ by adopting the notation in [4], with the correct homotopy type for an open subgroup $U$ of $G_{n}$ using that $G_{n} / U$ is finite. In a second step they defined $E_{n}^{d h G}$ for a closed subgroup $G$. Since $G_{n}$ is a $p$-adic analytic group, it is possible to find a sequence of open normal subgroups $G_{n}=U_{0} \supset U_{1} \supset \cdots$ whose intersection is the trivial subgroup. Then $E_{n}^{d h G}$ is defined as an appropriate homotopy colimit of the $E_{n}^{d h U_{i} G}$ 's. Moreover, for every closed subgroup $G$ of $G_{n}$, they provided a construction of a $K(n)_{*}$-local $E_{n}$-Adams spectral sequence converging to $\pi_{*}\left(E_{n}^{d h G}\right)$ whose $E_{2}$-term equals the desired continuous cohomology.

But since the argument of [8] did not explain in which sense $G_{n}$ acts continuously on $E_{n}$, the question remained how to view $E_{n}$ as an actual continuous $G_{n}$-spectrum and to find a natural framework for the continuous homotopy fixed point spectral sequence. The purpose of this paper is to give a new and complete answer to this question. 
A previous approach has been developed by Davis in [4] and by Behrens and Davis in [1] by studying discrete $G$-spectra. Davis used the idea of Devinatz and Hopkins to start with the homotopy fixed point spectrum $E_{n}^{d h U}$ of $[\mathbf{8}]$ for an open normal subgroup $U \subset G_{n}$ and defined a new spectrum $F_{n}:=\operatorname{colim}_{i} E_{n}^{d h U_{i}}$ where the $U_{i}$ run through a fixed sequence of open normal subgroups of $G_{n}$ as above. The $K(n)_{*}$-localization of $F_{n}$ is equivalent to $E_{n}$. One can regard the localization of $F_{n}$ as a continuous $G_{n}$-spectrum in the sense that it is the limit of a tower of discrete $G_{n}$-spectra. Then he defined systematically the homotopy fixed points for closed subgroups of $G_{n}$ and constructed a continuous homotopy fixed point spectral sequence. Furthermore, Davis developed a stable homotopy theory for discrete $G$-spectra, for an arbitrary profinite group $G$.

A different method has been used by Fausk. In [10], Fausk constructed a model structure for pro- $G$-spectra, where $G$ denotes a compact Hausdorff topological group, e.g., a profinite group. He also obtained results on homotopy fixed points and descent spectral sequences. The homotopy fixed point spectra of $[\mathbf{1 0}]$ are equivalent to those of [4] if $G$ has finite virtual cohomological dimension.

But the crucial point is that if one wants to use the methods of Davis or Fausk for Lubin-Tate spectra $E_{n}$, one first has to apply the construction of [8] for open subgroups and has to rewrite $E_{n}$ as the $K(n)_{*}$-localization of a suitable colimit of the $E_{n}^{d h U_{i}}$ 's as in [8]. Hence the above question of how to view $E_{n}$ as a continuous $G_{n}$-spectrum without using [8] for open subgroups of $G_{n}$ and of how to give a unified construction for all closed subgroups of $G_{n}$ without [8] still remained open.

The approach of the present paper provides a new unified natural construction of continuous homotopy fixed points for any closed subgroup independent of [8] and hence, in particular, also a new construction for open subgroups of $G_{n}$. The idea is straightforward. Since the homotopy groups $\pi_{t} E_{n}$ are not discrete but profinite $G_{n^{-}}$ modules, a natural guess would be to look for a profinite structure on $E_{n}$. And, in fact, there is one in the following sense. There is a model for $E_{n}$ that is built out of a sequence of simplicial profinite sets that carry a continuous $G_{n}$-action. Consequently, a natural setting to study the action of $G_{n}$ on $E_{n}$ is a suitable category of continuous profinite $G_{n}$-spectra.

Let us give a quick outline of the strategy and provide precise statements of the main results of this paper. A pointed profinite $G$-space is a simplicial object in the category of profinite sets with the limit topology and a continuous $G$-action together with a choice of basepoint. Pointed profinite $G$-spaces form a category $\hat{\mathcal{S}}_{* G}$ with levelwise basepoint preserving continuous $G$-equivariant maps as morphisms. A profinite $G$-spectrum $X$ is a sequence of pointed profinite $G$-spaces $X_{n}$ with maps $S^{1} \wedge X_{n} \rightarrow X_{n+1}$ for all $n$, where the simplicial circle $S^{1}$ is a simplicial finite set with trivial $G$-action. The homotopy theory of profinite $G$-spectra has been developed in $[\mathbf{2 4}]$ and we refer the reader to loc. cit. for any details.

The category $\operatorname{Sp}\left(\hat{\mathcal{S}}_{* G}\right)$ of profinite $G$-spectra is equipped with a natural stable model structure. The fibrant replacement functor $R_{G}$ in $\operatorname{Sp}\left(\hat{\mathcal{S}}_{* G}\right)$ enables us to give a natural definition for continuous homotopy fixed point spectra. In fact, the homotopy fixed point spectrum $X^{h G}$ of a profinite $G$-spectrum $X$ is defined as a continuous mapping spectrum $X^{h G}:=\operatorname{Map}_{G}\left(E G_{+}, R_{G} X\right)$ of $G$-equivariant and levelwise continuous maps in $\operatorname{Sp}\left(\hat{\mathcal{S}}_{* G}\right)$ (see Definitions 3.7 and 3.14). We will show that these homotopy 
fixed point spectra are equipped with a convergent spectral sequence

$$
H^{*}\left(G ; \pi_{*} X\right) \Rightarrow \pi_{*} X^{h G}
$$

whose $E_{2}$-terms are given by the continuous cohomology groups of $G$.

The striking advantage of studying profinite actions in the category of profinite spectra is that $G$ and its classifying space $E G$ yield natural objects in $\hat{\mathcal{S}}_{G}$. The homotopy fixed point spectral sequence is then obtained just as for a finite group by filtering $E G$ by its finite subskeleta. (But one should note that although $E G$ and $X$ are profinite, the function spectrum $\operatorname{Map}_{G}\left(E G_{+}, X\right)$ does not in general inherit a profinite structure, since, roughly speaking, the limit of $E G$ is turned into a colimit. Hence the homotopy groups of $X^{h G}$ are not profinite anymore in general.)

In order to be able to apply these techniques to the action of $G_{n}$ on the LubinTate spectrum $E_{n}$, we have to prove that we may consider $E_{n}$ as a profinite $G_{n^{-}}$ spectrum. The starting observation is that $E_{n}$ has a decomposition as a homotopy limit of spectra $\operatorname{holim}_{I} E_{n} \wedge M_{I}$, where the $M_{I}$ denote generalized Moore spectra corresponding to an inverse system of ideals $I$ in $B P_{*}$ (cf. [14]). These spectra have the important property that, for each such ideal $I$ and for every $t$, the homotopy group $\pi_{t}\left(E_{n} \wedge M_{I}\right)$ is finite (being trivial if $t$ is odd). The equivariant finite replacement functor for spectra with finite homotopy groups constructed in [24] provides a model of $E_{n} \wedge M_{I}$ in the category of profinite $G_{n}$-spectra. Taking the homotopy limit over all $I$ will yield a model of $E_{n}$ as a profinite $G_{n}$-spectrum. More precisely, we will show the following result.

Theorem 1.1. $E_{n}$ has a canonical model in the category of profinite $G_{n}$-spectra, i.e., there is a profinite $G_{n}$-spectrum $E_{n}^{\prime}$ and a $G_{n}$-equivariant isomorphism in the stable homotopy category $E_{n} \cong E_{n}^{\prime}$.

This allows us to apply the techniques developed for profinite $G$-spectra to $E_{n}$ and to prove the following theorem.

Theorem 1.2. Let $G$ be a closed subgroup of $G_{n}$.

(i) There is a $K(n)_{*}$-local continuous homotopy fixed point spectrum $E_{n}^{h G}$ of $E_{n}$ which is natural in $G$ and equivalent to the spectrum $E_{n}^{d h G}$ of [8]. In particular, there is an equivalence $E_{n}^{h G_{n}} \simeq E_{n}^{d h G_{n}} \simeq L_{K(n)} S^{0}$.

(ii) There is a natural strongly convergent continuous homotopy fixed point spectral sequence starting from continuous cohomology

$$
H^{*}\left(G ; \pi_{*} E_{n}\right) \Rightarrow \pi_{*} E_{n}^{h G}
$$

which is isomorphic to the $K(n)_{*}$-local $E_{n}$-Adams spectral sequence converging to $\pi_{*}\left(E_{n}^{d h G}\right)$.

This theorem restates some of the main results of $[\mathbf{8}]$ in the setting of profinite $G$-spectra. The point is that it has a conceptually simpler proof. While in $[8]$ the special properties of $G_{n}$ and $E_{n}$ have been taken advantage of to define homotopy fixed points, the methods we develop for the construction of homotopy fixed point spectra and for the construction of the homotopy descent spectral sequence in Theorem 1.2 are general and work for any continuous action of a profinite group on any profinite spectrum. In particular, the construction of $E_{n}^{h G}$ is the same for all closed 
(or open) subgroups. Nevertheless, we remark that our construction provides no algebraic structure on $E_{n}^{h G}$, whereas $[8]$ shows that $E_{n}^{d h G}$ is a commutative $\mathbb{S}^{0}$-algebra. It is an advantage of the work of Behrens and Davis in [1] that they are also able to consider additional algebraic structures.

For the proof of Theorem 1.2 we will actually compare our construction with the construction of $[\mathbf{4}]$ and $[\mathbf{1}]$. This shortcut has been suggested by the anonymous referee and we gratefully acknowledge his contribution and generosity. The proof also answers the question of how all the available homotopy fixed point spectra and descent spectral sequences are related to each other. Denoting the continuous homotopy fixed points of Davis [4] by $E_{n}^{h^{\prime} G}$ we will show that there is an equivalence of spectra

$$
E_{n}^{h G} \simeq E_{n}^{h^{\prime} G}
$$

Moreover, this equivalence is equipped with a map between descent spectral sequences converging to $\pi_{*}\left(E_{n}^{h G}\right)$ and $\pi_{*}\left(E_{n}^{h^{\prime} G}\right)$ respectively which is an isomorphism from the $E_{2}$-terms on. To deduce the statement of the theorem we then use that the work of Davis [4] and Behrens-Davis [1] shows that the homotopy fixed point spectrum $E_{n}^{h^{\prime} G}$ is equivalent to the spectrum $E_{n}^{d h G}$ of Devinatz-Hopkins [8] and that the descent spectral sequence converging to $\pi_{*}\left(E_{n}^{h^{\prime} G}\right)$ is isomorphic from the $E_{2}$-term on to the $K(n)_{*}$-local $E_{n}$-Adams spectral sequence converging to $\pi_{*}\left(E_{n}^{d h G}\right)$.

\section{Acknowledgements}

The starting point of this project was a question by Dan Isaksen if the profinite spectra of $[\mathbf{2 3}]$ fit in the theory of Lubin-Tate spectra. We would like to thank him very much for this generous hint. We are very grateful to Mike Hopkins for very helpful discussions. We would like to thank Daniel Davis for numerous comments. Finally, we would like to thank the anonymous referee for his suggestions and contributions.

\section{A short review of profinite $G$-spectra}

For the convenience of the reader, we recall some notation and results about profinite $G$-spectra that we will use throughout the paper. For any details and proofs of the results cited we refer the reader to $[\mathbf{2 4}]$ and the references given therein.

\subsection{Profinite $G$-spaces}

Let $\mathcal{E}$ denote the category of sets and let $\hat{\mathcal{E}}$ be the category of compact Hausdorff and totally disconnected topological spaces, or equivalently the category of profinite sets with the profinite topology. The forgetful functor $\hat{\mathcal{E}} \rightarrow \mathcal{E}$ admits a left adjoint $(\hat{)}): \mathcal{E} \rightarrow \hat{\mathcal{E}}$ which is called profinite completion.

We denote by $\hat{\mathcal{S}}$ (resp., $\mathcal{S}$ ) the category of simplicial profinite sets (resp., simplicial sets). The objects of $\hat{\mathcal{S}}$ (resp. $\mathcal{S}$ ) will be called profinite spaces (resp., spaces). The profinite completion of sets induces levelwise a functor $(\hat{.}): \mathcal{S} \rightarrow \hat{\mathcal{S}}$, which is also called profinite completion. It is left adjoint to the forgetful functor $|\cdot|: \hat{\mathcal{S}} \rightarrow \mathcal{S}$ which sends a profinite space to its underlying simplicial set.

Let $G$ be a profinite group. Let $S$ be a profinite set on which $G$ acts continuously, i.e., the group $G$ is acting on $S$ via a continuous map $\mu: G \times S \rightarrow S$. In this situation 
we say that $S$ is a profinite $G$-set. The morphisms between profinite $G$-sets are $G$ equivariant continuous maps. Let $\hat{\mathcal{S}}_{G}$ be the category of simplicial objects in the category of profinite $G$-sets. We call the objects of $\hat{\mathcal{S}}_{G}$ profinite $G$-spaces.

Example 2.1. Important examples are the classifying spaces of $G$. The simplicial set $E G$, whose set of $n$-simplices is $E G_{n}=G^{n+1}$, the $(n+1)$-fold product of $G$ with a free and continuous action of $G$ in each dimension, is a profinite $G$-space. The quotient $B G=E G / G$ is the profinite space whose set of $n$-simplices is $B G_{n}=G^{n}$, the $n$-fold product of $G$. The face and degeneracy maps of $E G$ and $B G$ will be discussed in the next section.

Let $\hat{\mathcal{S}}_{* G}$ be the category of pointed profinite $G$-spaces. The objects of $\hat{\mathcal{S}}_{* G}$ are profinite $G$-spaces that are equipped with a basepoint that is fixed under $G$. The morphisms in $\hat{\mathcal{S}}_{* G}$ are the morphisms of profinite $G$-spaces that preserve the basepoints. If $X$ is a profinite $G$-space, we denote by $X_{+}$the pointed profinite $G$-space consisting of $X$ with a disjoint fixed basepoint.

Let $X$ and $Y$ be pointed profinite $G$-spaces. The smash product $X \wedge Y$ is again a pointed profinite $G$-space on which $G$ acts via the diagonal action. For $X, Y \in \hat{\mathcal{S}}_{* G}$, the mapping space $\operatorname{map}_{\hat{\mathcal{S}}_{* G}}(X, Y)$ is defined as the simplicial set whose set of $n$-simplices is given as the set of maps $\operatorname{map}_{\hat{\mathcal{S}}_{* G}}(X, Y)_{n}=\operatorname{Hom}_{\hat{\mathcal{S}}_{* G}}\left(X \wedge \Delta[n]_{+}, Y\right)$ where $\Delta[n]_{+}$is considered as a pointed profinite $G$-space with trivial $G$-action.

Let $K$ be a finite simplicial set, i.e., a simplicial set which has only finitely many nondegenerate simplices. This implies in particular that $K$ is a simplicial finite set. Let $X$ be a pointed profinite $G$-space. The tensor object $X \otimes K \in \hat{\mathcal{S}}_{* G}$ is defined as the smash product $X \wedge K_{+}$where $K_{+}$is considered as a pointed profinite $G$-space with trivial $G$-action. The function object in $\hat{\mathcal{S}}_{* G}$ is defined as the pointed profinite $G$-space $\operatorname{hom}_{\hat{\mathcal{S}}_{* G}}(K, X) \in \hat{\mathcal{S}}_{* G}$ whose set of $n$-simplices is given by the profinite set of maps

$$
\operatorname{hom}_{\hat{\mathcal{S}}_{* G}}(K, X)_{n}=\operatorname{Hom}_{\hat{\mathcal{S}}_{*}}\left(K_{+} \wedge \Delta[n]_{+}, X\right)
$$

on which $G$ acts continuously via its action on the target $X$.

If $K$ is an arbitrary simplicial set, isomorphic to the filtered colimit $\operatorname{colim}_{\alpha} K_{\alpha}$ of its finite simplicial subsets $K_{\alpha}$, and $X$ a pointed profinite $G$-space, we define the tensor object $X \otimes K$ to be the colimit in $\hat{\mathcal{S}}_{* G}$ of the pointed profinite $G$-spaces $X \otimes K_{\alpha}$. The function object $\operatorname{hom}_{\hat{\mathcal{S}}_{* G}}(K, X)$ is defined to be the limit in $\hat{\mathcal{S}}_{* G}$ of the pointed profinite $G$-spaces $\operatorname{hom}_{\hat{\mathcal{S}}_{* G}}\left(K_{\alpha}, X\right)$.

If the simplicial set $K$ is already equipped with a basepoint and $X$ is a pointed profinite $G$-space, we also denote by $\operatorname{hom}_{\hat{\mathcal{S}}_{* G}}(K, X) \in \hat{\mathcal{S}}_{* G}$ the pointed profinite $G$ space whose set of $n$-simplices is given by the profinite set of maps

$$
\operatorname{hom}_{\hat{\mathcal{S}}_{* G}}(K, X)_{n}=\lim _{\alpha} \operatorname{Hom}_{\hat{\mathcal{S}}_{*}}\left(K_{\alpha} \wedge \Delta[n]_{+}, X\right) .
$$

Example 2.2. Let $S^{1}$ be the simplicial circle, i.e., the quotient $S^{1}=\Delta[1] / \partial \Delta[1]$ of the standard simplex $\Delta[1]$ by its boundary. The pointed simplicial set $S^{1}$ is finite in each degree, i.e., it is a simplicial finite set and hence also an object in $\hat{\mathcal{S}}_{*}$. We consider $S^{1}$ as a pointed simplicial finite set with trivial $G$-action. Taking the smash product with $S^{1}$ defines a functor $\hat{\mathcal{S}}_{* G} \rightarrow \hat{\mathcal{S}}_{* G}, X \mapsto S^{1} \wedge X$. It is left adjoint to the functor $\hat{\mathcal{S}}_{* G} \rightarrow \hat{\mathcal{S}}_{* G}$ defined by sending a pointed profinite $G$-space $X$ to the function object 
$\operatorname{hom}_{\hat{\mathcal{S}}_{* G}}\left(S^{1}, X\right)=: \Omega X$ in $\hat{\mathcal{S}}_{* G}$. Note that the underlying pointed simplicial set $|\Omega X|$ is canonically isomorphic to the pointed simplicial set $\Omega|X|=\operatorname{map}_{\mathcal{S}_{*}}\left(S^{1},|X|\right)$.

The categories $\hat{\mathcal{S}}, \hat{\mathcal{S}}_{*}, \hat{\mathcal{S}}_{G}$ and $\hat{\mathcal{S}}_{* G}$ can be equipped with simplicial model structures.

\subsection{Profinite $G$-spectra}

A profinite spectrum $X$ consists of a sequence of pointed profinite spaces $X_{n} \in \hat{\mathcal{S}}_{*}$ and maps $\sigma_{n}: S^{1} \wedge X_{n} \rightarrow X_{n+1}$ in $\hat{\mathcal{S}}_{*}$ for $n \geqslant 0$. A morphism $f: X \rightarrow Y$ of profinite spectra consists of maps $f_{n}: X_{n} \rightarrow Y_{n}$ in $\hat{\mathcal{S}}_{*}$ for $n \geqslant 0$ such that $\sigma_{n}\left(1 \wedge f_{n}\right)=f_{n+1} \sigma_{n}$. We denote by $\operatorname{Sp}\left(\hat{\mathcal{S}}_{*}\right)$ the corresponding category of profinite spectra.

There is a stable model structure on $\operatorname{Sp}\left(\hat{\mathcal{S}}_{*}\right)$. The associated stable homotopy category is denoted by $\hat{\mathcal{S H}}$. For a profinite spectrum $X$, let $R X$ denote a functorial fibrant replacement of $X$ in the stable model structure on $\operatorname{Sp}\left(\hat{\mathcal{S}}_{*}\right)$ and, for an integer $n$, let $\mathbb{S}^{n}$ be the $n$th suspension of the sphere spectrum considered as a profinite spectrum. The $n$th stable homotopy group $\pi_{n} X$ of the profinite spectrum $X$ is defined to be the abelian group

$$
\pi_{n} X:=\operatorname{Hom}_{\mathcal{S H \mathcal { H }}}\left(\mathbb{S}^{n}, R X\right) .
$$

Definition 2.3. Let $G$ be a profinite group. We consider $S^{1}$ as a simplicial finite set with trivial $G$-action. A profinite $G$-spectrum $X$ is a sequence of pointed profinite $G$-spaces $\left\{X_{n}\right\}$ together with maps $S^{1} \wedge X_{n} \rightarrow X_{n+1}$ of pointed profinite $G$-spaces for each $n \geqslant 0$. A map of profinite $G$-spectra $X \rightarrow Y$ is a collection of maps $X_{n} \rightarrow Y_{n}$ in $\hat{\mathcal{S}}_{* G}$ compatible with the structure maps of $X$ and $Y$. We denote the category of profinite $G$-spectra by $\operatorname{Sp}\left(\hat{\mathcal{S}}_{* G}\right)$.

Profinite $G$-spectra form a simplicial category. For $X, Y \in \operatorname{Sp}\left(\hat{\mathcal{S}}_{* G}\right)$, the mapping space $\operatorname{map}_{\mathrm{Sp}\left(\hat{\mathcal{S}}_{* G}\right)}(X, Y)$ is defined as the simplicial set whose set of $n$-simplices is given as the set of maps

$$
\operatorname{map}_{\mathrm{Sp}\left(\hat{\mathcal{S}}_{* G}\right)}(X, Y)_{n}=\operatorname{Hom}_{\mathrm{Sp}\left(\hat{\mathcal{S}}_{* G}\right)}\left(X \wedge \Delta[n]_{+}, Y\right)
$$

where the smash product is defined levelwise.

Let $K$ be a simplicial set and $X$ a profinite $G$-spectrum. We define the tensor object $X \otimes K \in \operatorname{Sp}\left(\hat{\mathcal{S}}_{* G}\right)$ as the profinite $G$-spectrum whose $n$th pointed profinite $G$ space is $X_{n} \wedge K_{+}$. The function object in $\operatorname{Sp}\left(\hat{\mathcal{S}}_{* G}\right)$ is defined as the profinite spectrum $\operatorname{hom}_{\operatorname{Sp}\left(\hat{\mathcal{S}}_{* G}\right)}(K, X) \in \operatorname{Sp}\left(\hat{\mathcal{S}}_{* G}\right)$ whose $n$th pointed profinite $G$-space is given by

$$
\operatorname{hom}_{\mathrm{Sp}\left(\hat{\mathcal{S}}_{* G}\right)}(K, X)_{n}=\operatorname{hom}_{\hat{\mathcal{S}}_{* G}}\left(K, X_{n}\right) \text {. }
$$

The structure map of $\operatorname{hom}_{\mathrm{Sp}\left(\hat{\mathcal{S}}_{* G}\right)}(K, X)$ is the adjoint of the $G$-equivariant map

$$
\operatorname{hom}_{\hat{\mathcal{S}}_{* G}}\left(K, X_{n}\right) \rightarrow \operatorname{hom}_{\hat{\mathcal{S}}_{* G}}\left(K, \Omega X_{n+1}\right) \cong \Omega\left(\operatorname{hom}_{\hat{\mathcal{S}}_{* G}}\left(K, X_{n+1}\right)\right) .
$$

The stable model structure on $\operatorname{Sp}\left(\hat{\mathcal{S}}_{* G}\right)$ is constructed in two steps. First one proves that there is a projective model structure. A map $f$ in $\operatorname{Sp}\left(\hat{\mathcal{S}}_{* G}\right)$ is a projective weak equivalence (projective fibration) if each map $f_{n}$ is a weak equivalence (fibration) in $\hat{\mathcal{S}}_{* G}$. A map $i$ is a projective cofibration if it has the left lifting property with respect to all projective trivial fibrations. In a second step, the projective model structure is localized at $\Omega$-spectra. 
Definition 2.4. A profinite $G$-spectrum $E \in \operatorname{Sp}\left(\hat{\mathcal{S}}_{* G}\right)$ is called an $\Omega$-spectrum if each $E_{n}$ is fibrant in $\hat{\mathcal{S}}_{* G}$ and the adjoint structure maps $E_{n} \rightarrow \Omega E_{n+1}$ are weak equivalences in $\hat{\mathcal{S}}_{* G}$ for all $n$. A map $f: X \rightarrow Y$ of profinite $G$-spectra is called a (stable) equivalence if any projective cofibrant replacement $Q_{G} f: Q_{G} X \rightarrow Q_{G} Y$ in $\operatorname{Sp}\left(\hat{\mathcal{S}}_{* G}\right)$ induces a weak equivalence of mapping spaces

$$
\operatorname{map}_{\mathrm{Sp}\left(\hat{\mathcal{S}}_{* G}\right)}\left(Q_{G} Y, E\right) \rightarrow \operatorname{map}_{\mathrm{Sp}\left(\hat{\mathcal{S}}_{* G}\right)}\left(Q_{G} X, E\right)
$$

for every $\Omega$-spectrum $E$ in $\operatorname{Sp}\left(\hat{\mathcal{S}}_{* G}\right)$.

Theorem 2.5. There is a stable simplicial model structure on $\operatorname{Sp}\left(\hat{\mathcal{S}}_{* G}\right)$ for which the weak equivalences are the stable equivalences and the fibrant profinite $G$-spectra are exactly the $\Omega$-spectra of Definition 2.4. A map $f: X \rightarrow Y$ in $\operatorname{Sp}\left(\hat{\mathcal{S}}_{* G}\right)$ between $\Omega$ spectra is a stable equivalence if and only if each $f_{n}: X_{n} \rightarrow Y_{n}$ is a weak equivalence in $\hat{\mathcal{S}}_{* G}$. We denote its homotopy category by $\hat{\mathcal{S H}}_{G}$. The stable homotopy groups of an $\Omega$-spectrum in $\operatorname{Sp}\left(\hat{\mathcal{S}}_{* G}\right)$ (as defined in (3)) have a canonical structure as profinite G-modules.

Proposition 2.6. (1) Let $K$ be a closed subgroup of the profinite group $G$. If $X$ is an $\Omega$-spectrum in $\operatorname{Sp}\left(\hat{\mathcal{S}}_{* G}\right)$, then its restriction to a profinite $K$-spectrum is also an $\Omega$-spectrum in $\operatorname{Sp}\left(\hat{\mathcal{S}}_{* K}\right)$.

(2) Let $\operatorname{Sp}\left(\mathcal{S}_{*}\right)$ be the category of Bousfield-Friedlander spectra [2]. The composition of forgetful functors $\operatorname{Sp}\left(\hat{\mathcal{S}}_{* G}\right) \rightarrow \operatorname{Sp}\left(\hat{\mathcal{S}}_{*}\right) \rightarrow \operatorname{Sp}\left(\mathcal{S}_{*}\right)$, which we also denote by $|\cdot|$, sends $\Omega$-spectra to $\Omega$-spectra and preserves stable equivalences between $\Omega$-spectra.

(3) Let $X$ be an $\Omega$-spectrum in $\operatorname{Sp}\left(\hat{\mathcal{S}}_{* G}\right)$. The underlying groups of the profinite homotopy groups of $X$ are isomorphic to the stable homotopy groups of the underlying spectrum $|X|$ in $\operatorname{Sp}\left(\mathcal{S}_{*}\right)$.

Let $I$ be a small category and let $X(-)$ be a functor from $I$ to the full subcategory of $\Omega$-spectra in $\operatorname{Sp}\left(\hat{\mathcal{S}}_{* G}\right)$. For each $n \geqslant 0$ and each $i \in I$, the pointed profinite $G$-space $X_{n}(i):=X(i)_{n}$ is fibrant. This implies that for every $n \geqslant 0$, the homotopy limit $\operatorname{holim}_{i \in I} X_{n}(i)$ in $\hat{\mathcal{S}}_{* G}$ is a fibrant pointed profinite $G$-space and there is a natural isomorphism $\operatorname{holim}_{i \in I} \Omega X_{n}(i) \cong \Omega \operatorname{holim}_{i \in I} X_{n}(i)$ in $\hat{\mathcal{S}}_{* G}$ (see [24]). Since each $X(i)$ is an $\Omega$-spectrum in $\operatorname{Sp}\left(\hat{\mathcal{S}}_{* G}\right)$ and since holim $\operatorname{lif}_{i \in I}$ preserves weak equivalences between fibrant objects, we obtain for each $n$ a weak equivalence in $\hat{\mathcal{S}}_{* G}$

$$
\operatorname{holim}_{i \in I} X_{n}(i) \stackrel{\sim}{\rightarrow} \operatorname{holim}_{i \in I} \Omega X_{n}(i) \cong \Omega \operatorname{holim}_{i \in I} X_{n}(i) .
$$

Hence together with the adjoints of these maps as structure maps the sequence $\operatorname{holim}_{i \in I} X_{n}(i)$ of fibrant pointed profinite $G$-spaces defines an $\Omega$-spectrum in $\operatorname{Sp}\left(\hat{\mathcal{S}}_{* G}\right)$ that we denote by $\operatorname{holim}_{i \in I} X(i)$ and call the homotopy limit of the diagram $X(-)$.

Finally, a crucial result of $[\mathbf{2 4}]$ is the construction of concrete models in the category of profinite $G$-spectra for ordinary $G$-spectra with finite homotopy groups.

A profinite group $G$ is called strongly complete if every subgroup of finite index is open in $G$. A consequence of this property is that for a strongly complete profinite group $G$, every finite set $S$ with a $G$-action is a continuous discrete $G$-set. The profinite completion of an abstract group is strongly complete. But in general there are subgroups of finite index which are not open in the given topology. It is the important result of Nikolov and Segal $[\mathbf{2 2}]$ that every finitely generated profinite group is 
strongly complete. The most important example for us is provided by the extended Morava stabilizer group $G_{n}$ and all of its closed subgroups (see e.g., [4], p. 330).

Theorem 2.7. Let $G$ be a strongly complete profinite group. Let $X \in \operatorname{Sp}\left(\mathcal{S}_{*}\right)$ be a spectrum such that each space $X_{n}$ is a pointed $G$-space and the $G$-actions are compatible with the structure maps. We assume that the homotopy groups of $X$ are all finite groups. Then there is a G-equivariant map

$$
\varphi^{s}: X \rightarrow F_{G}^{s} X
$$

of spectra from $X$ to a profinite $G$-spectrum $F_{G}^{s} X$ built of simplicial finite discrete $G$ sets such that $F_{G}^{s} X$ is fibrant in $\operatorname{Sp}\left(\hat{\mathcal{S}}_{* G}\right)$ and $\varphi^{s}$ is a stable equivalence of underlying spectra. In particular, $\varphi^{s}$ induces a $G$-equivariant isomorphism $\pi_{*} X \cong \pi_{*}\left|F_{G}^{s} X\right|$ of the homotopy groups of underlying spectra.

The assignment $X \mapsto F_{G}^{s} X$ is functorial in the sense that given a $G$-equivariant map $h: X \rightarrow Y$ between $G$-spectra whose homotopy groups are finite, there is a map $F_{G}^{s}(h)$ in $\operatorname{Sp}\left(\hat{\mathcal{S}}_{* G}\right)$ such that the following diagram of underlying spectra commutes

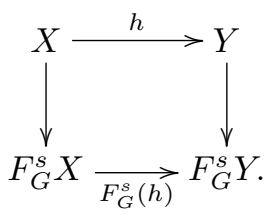

\section{Homotopy fixed point spectra}

\subsection{Continuous equivariant mapping spectra}

Let $Y$ be a profinite space and $W$ be a pointed profinite space. The functor $\hat{\mathcal{S}} \rightarrow \hat{\mathcal{S}}_{*}$, $Y \rightarrow Y_{+}$, defined by adding a disjoint basepoint, is the left adjoint of the functor that forgets the basepoint. Hence there is a natural isomorphism of simplicial sets

$$
\operatorname{map}_{\hat{\mathcal{S}}_{*}}\left(Y_{+}, W\right) \cong \operatorname{map}_{\hat{\mathcal{S}}}(Y, W) .
$$

The spaces on both sides of (4) are pointed by the map that factors through $* \rightarrow W$. Hence (4) is in fact an isomorphism of pointed simplicial sets. We will use the notation $\operatorname{Map}(Y, W)$ for the pointed simplicial set $\operatorname{map}_{\hat{\mathcal{S}}_{*}}\left(Y_{+}, W\right)$ together with its basepoint $Y_{+} \rightarrow * \rightarrow W$. This defines a functor

$$
\operatorname{Map}(-,-): \hat{\mathcal{S}}^{\mathrm{op}} \times \hat{\mathcal{S}}_{*} \rightarrow \mathcal{S}_{*} .
$$

Definition 3.1. Let $G$ be a profinite group. Let $Y$ be a profinite $G$-space and $W$ be a pointed profinite $G$-space. We define $\operatorname{Map}_{G}(Y, W)$ to be the pointed simplicial set $\operatorname{map}_{\hat{\mathcal{S}}_{* G}}\left(Y_{+}, W\right)$ pointed by the map $Y_{+} \rightarrow * \rightarrow W$. This defines a functor

$$
\operatorname{Map}_{G}(-,-): \hat{\mathcal{S}}_{G}^{\mathrm{op}} \times \hat{\mathcal{S}}_{* G} \rightarrow \mathcal{S}_{*} .
$$

When $Y$ is a profinite $G$-space and $W$ is a pointed profinite $G$-space, we can equip the pointed simplicial set $\operatorname{Map}(Y, W)$ with a $G$-action by $(g f)(y):=g f\left(g^{-1} y\right)$. With this $G$-action on $\operatorname{Map}(Y, W), \operatorname{Map}_{G}(Y, W)$ is the pointed space of $G$-fixed points of the pointed space $\operatorname{Map}(Y, W)$. 
Lemma 3.2. Let $i: A \rightarrow B$ be a cofibration of profinite $G$-spaces and $W$ be a fibrant pointed profinite $G$-space. Then

$$
\operatorname{Map}_{G}(i, W): \operatorname{Map}_{G}(B, W) \rightarrow \operatorname{Map}_{G}(A, W)
$$

is a fibration of pointed simplicial sets.

Proof. Since $W$ is fibrant and $\hat{\mathcal{S}}_{* G}$ is a simplicial model category, the map

$$
\operatorname{map}_{\hat{\mathcal{S}}_{* G}}\left(B_{+}, W\right) \rightarrow \operatorname{map}_{\hat{\mathcal{S}}_{* G}}\left(A_{+}, W\right)
$$

is a fibration of simplicial sets. Thus $\operatorname{Map}_{G}(i, W)$ is a fibration of pointed spaces.

Lemma 3.3. Let $Y$ be a cofibrant profinite $G$-space and $f: V \rightarrow W$ be a fibration between pointed profinite $G$-spaces. Then

$$
\operatorname{Map}_{G}(Y, f): \operatorname{Map}_{G}(Y, V) \rightarrow \operatorname{Map}_{G}(Y, W)
$$

is a fibration of pointed simplicial sets.

Proof. Since $Y_{+}$is cofibrant and $\hat{\mathcal{S}}_{* G}$ is a simplicial model category, the map

$$
\operatorname{map}_{\hat{\mathcal{S}}_{* G}}\left(Y_{+}, V\right) \rightarrow \operatorname{map}_{\hat{\mathcal{S}}_{* G}}\left(Y_{+}, W\right)
$$

is a fibration of simplicial sets. Thus $\operatorname{Map}_{G}(Y, f)$ is a fibration of pointed spaces.

Lemma 3.4. Let $Y$ be a cofibrant profinite $G$-space, $f: V \rightarrow W$ be a weak equivalence between fibrant pointed profinite $G$-spaces. Then

$$
\operatorname{Map}_{G}(Y, f): \operatorname{Map}_{G}(Y, V) \rightarrow \operatorname{Map}_{G}(Y, W)
$$

is a weak equivalence of fibrant pointed simplicial sets.

Proof. The profinite space $Y$ is a cofibrant object in $\hat{\mathcal{S}}_{G}$ and $V$ and $W$ are fibrant objects in $\hat{\mathcal{S}}_{* G}$ by assumption. Since $\hat{\mathcal{S}}_{* G}$ is a simplicial model category, the induced map $\operatorname{map}_{\hat{\mathcal{S}}_{* G}}\left(Y_{+}, V\right) \rightarrow \operatorname{map}_{\hat{\mathcal{S}}_{* G}}\left(Y_{+}, W\right)$ is a weak equivalence of fibrant simplicial sets. Hence the pointed map $\operatorname{Map}_{G}(Y, f)$ is a weak equivalence of fibrant pointed spaces.

Lemma 3.5. Let $Y$ be a cofibrant profinite $G$-space. Let $X(-): I \rightarrow \hat{\mathcal{S}}_{* G}$ be a small diagram of fibrant pointed profinite $G$-spaces. Then there is a natural isomorphism of fibrant pointed simplicial sets

$$
\operatorname{Map}_{G}\left(Y, \operatorname{holim}_{i \in I} X(i)\right) \cong \operatorname{holim}_{i \in I} \operatorname{Map}_{G}(Y, X(i))
$$

where $\operatorname{holim}_{i \in I} \operatorname{Map}_{G}(Y, X(i))$ denotes the homotopy limit in $\mathcal{S}_{*}$ of the small diagram $\operatorname{Map}_{G}(Y, X(-)): I \rightarrow \mathcal{S}_{*}$ of pointed simplicial sets. In particular, $\operatorname{Map}_{G}(Y,-)$ preserves homotopy fibers.

Proof. Since $Y$ is a cofibrant object in $\hat{\mathcal{S}}_{G}, \operatorname{Map}_{G}(Y,-)$ preserves fibrations. Moreover, being a right adjoint functor, $\operatorname{Map}_{G}(Y,-)$ preserves products and limits and sends cotensors to cotensors in the sense that there is a natural isomorphism of pointed simplicial sets

$$
\operatorname{Map}_{G}\left(Y, \operatorname{hom}_{\hat{\mathcal{S}}_{* G}}(K, W)\right) \cong \operatorname{hom}_{\mathcal{S}_{*}}\left(K, \operatorname{Map}_{G}(Y, W)\right)
$$

for every simplicial set $K$, where the right hand space is equal to the pointed space $\operatorname{map}_{\mathcal{S}_{*}}\left(K, \operatorname{Map}_{G}(Y, W)\right)$ whose basepoint is the map having constant image the basepoint of $\operatorname{Map}_{G}(Y, W)$. Thus $\operatorname{Map}_{G}(Y,-)$ sends the equalizer of the diagram of pointed 
profinite $G$-spaces

$$
\prod_{i \in I} \operatorname{hom}_{\hat{\mathcal{S}}_{* G}}(B(I / i), X(i)) \rightrightarrows \prod_{\alpha: i \rightarrow i^{\prime} \in I} \operatorname{hom}_{\hat{\mathcal{S}}_{* G}}\left(B(I / i), X\left(i^{\prime}\right)\right),
$$

which is by definition holim $i \in I X(i)$, to the equalizer in $\mathcal{S}_{*}$ of the diagram of pointed spaces

$$
\prod_{i \in I} \operatorname{hom}_{\mathcal{S}_{*}}\left(B(I / i), \operatorname{Map}_{G}(Y, X(i))\right) \rightrightarrows \prod_{\alpha: i \rightarrow i^{\prime} \in I} \operatorname{hom}_{\mathcal{S}_{*}}\left(B(I / i), \operatorname{Map}_{G}\left(Y, X\left(i^{\prime}\right)\right)\right) .
$$

Since the equalizer of the last diagram is by definition $\operatorname{holim}_{i \in I} \operatorname{Map}_{G}(Y, X(i))$, this proves the assertion. The statement on homotopy fibers is a special case of the first assertion.

Now we turn our attention to continuous mapping spectra.

Definition 3.6. For a profinite space $Y$ and a profinite spectrum $X$, we denote by $\operatorname{Map}(Y, X)$ the spectrum whose $n$th space is given by the pointed simplicial set $\operatorname{Map}\left(Y, X_{n}\right)$. This defines a functor

$$
\operatorname{Map}(-,-): \hat{\mathcal{S}}^{\mathrm{op}} \times \operatorname{Sp}\left(\hat{\mathcal{S}}_{*}\right) \rightarrow \operatorname{Sp}\left(\mathcal{S}_{*}\right) .
$$

Definition 3.7. Let $Y$ be a profinite $G$-space and $X$ a profinite $G$-spectrum. We define $\operatorname{Map}_{G}(Y, X)$ to be the spectrum whose $n$th space is given by the pointed simplicial set $\operatorname{Map}_{G}\left(Y, X_{n}\right)$ defined in Definition 3.1. The structure maps are defined as follows. The compatibilities of mapping spaces and cotensors provide an isomorphism

$$
\operatorname{Map}_{G}\left(Y, \operatorname{hom}_{\hat{\mathcal{S}}_{*}}\left(S^{1}, X_{n}\right)\right) \cong \operatorname{hom}_{\mathcal{S}_{*}}\left(S^{1}, \operatorname{Map}_{G}\left(Y, X_{n}\right)\right) \text {. }
$$

The space on the left hand side is $\operatorname{Map}_{G}\left(Y, \Omega\left(X_{n}\right)\right)$ and the space on the right hand side is $\Omega\left(\operatorname{Map}_{G}\left(Y, X_{n}\right)\right)$. Hence the map $X_{n} \rightarrow \Omega X_{n+1}$ defines a map

$$
\operatorname{Map}_{G}\left(Y, X_{n}\right) \rightarrow \operatorname{Map}_{G}\left(Y, \Omega X_{n+1}\right) \cong \Omega\left(\operatorname{Map}_{G}\left(Y, X_{n+1}\right)\right) .
$$

This provides a functor

$$
\operatorname{Map}_{G}(-,-): \hat{\mathcal{S}}_{G}^{\mathrm{op}} \times \operatorname{Sp}\left(\hat{\mathcal{S}}_{* G}\right) \rightarrow \operatorname{Sp}\left(\mathcal{S}_{*}\right) .
$$

Remark 3.8. Let $Y$ be a profinite $G$-space and $X$ a profinite $G$-spectrum. If we equip again $\operatorname{Map}\left(Y, X_{n}\right)$ with the above $G$-action, then we can consider $\operatorname{Map}(Y, X)$ as a spectrum which is built out of pointed spaces with a $G$-action and $\operatorname{Map}_{G}(Y, X)$ is the spectrum of fixed points of $\operatorname{Map}(Y, X)$.

Lemma 3.9. Let $i: A \rightarrow B$ be a cofibration of profinite $G$-spaces and $X$ be an $\Omega$ spectrum in $\operatorname{Sp}\left(\hat{\mathcal{S}}_{* G}\right)$. Then

$$
\operatorname{Map}_{G}(i, X): \operatorname{Map}_{G}(B, X) \rightarrow \operatorname{Map}_{G}(A, X)
$$

is a projective (i.e., levelwise) fibration of Bousfield-Friedlander spectra.

Proof. This follows from Lemma 3.2 and the definition of projective fibrations.

Lemma 3.10. Let $Y$ be a cofibrant profinite $G$-space. If $X$ is an $\Omega$-spectrum in $\operatorname{Sp}\left(\hat{\mathcal{S}}_{* G}\right)$, then $\operatorname{Map}_{G}(Y, X)$ is an $\Omega$-spectrum in $\operatorname{Sp}\left(\mathcal{S}_{*}\right)$. 
Proof. This follows from Lemma 3.4 and the definition of the structure maps of $\operatorname{Map}_{G}(Y, X)$ in (5).

Lemma 3.11. Let $Y$ be a cofibrant profinite $G$-space. The functor $\operatorname{Map}_{G}(Y,-)$ sends stable equivalences between profinite $\Omega$-spectra in $\operatorname{Sp}\left(\hat{\mathcal{S}}_{* G}\right)$ to stable equivalences between $\Omega$-spectra in $\operatorname{Sp}\left(\mathcal{S}_{*}\right)$.

Proof. Let $f: X \rightarrow X^{\prime}$ be a stable equivalence between profinite $\Omega$-spectra in $\operatorname{Sp}\left(\hat{\mathcal{S}}_{* G}\right)$. By Lemma $3.10, \operatorname{Map}_{G}(Y, X)$ and $\operatorname{Map}_{G}\left(Y, X^{\prime}\right)$ are $\Omega$-spectra in $\operatorname{Sp}\left(\mathcal{S}_{*}\right)$. Since stable equivalences between $\Omega$-spectra in $\operatorname{Sp}\left(\hat{\mathcal{S}}_{* G}\right)$ and $\operatorname{Sp}\left(\mathcal{S}_{*}\right)$ are exactly the projective (i.e., levelwise) equivalences, the assertion now follows from Lemma 3.4.

Proposition 3.12. Let $Y$ be a cofibrant profinite $G$-space. Let $X(-): I \rightarrow \operatorname{Sp}\left(\hat{\mathcal{S}}_{* G}\right)$ be a small diagram of $\Omega$-spectra in $\operatorname{Sp}\left(\hat{\mathcal{S}}_{* G}\right)$. Then there is a natural isomorphism of $\Omega$-spectra in $\operatorname{Sp}\left(\mathcal{S}_{*}\right)$

$$
\operatorname{Map}_{G}\left(Y, \operatorname{holim}_{i \in I} X(i)\right) \cong \operatorname{holim}_{i \in I} \operatorname{Map}_{G}(Y, X(i))
$$

where $\operatorname{holim}_{i \in I} \operatorname{Map}_{G}(Y, X(i))$ denotes the homotopy limit in $\operatorname{Sp}\left(\mathcal{S}_{*}\right)$ of the small diagram $\operatorname{Map}_{G}(Y, X(-)): I \rightarrow \operatorname{Sp}\left(\mathcal{S}_{*}\right)$ of $\Omega$-spectra in $\operatorname{Sp}\left(\mathcal{S}_{*}\right)$.

In particular, the functor $\operatorname{Map}_{G}(Y,-)$ preserves homotopy fibers of maps between $\Omega$-spectra.

Proof. Since $\operatorname{Map}_{G}(Y,-)$ and homotopy limits are defined levelwise, the first assertion follows from Lemma 3.5. The second assertion is a special case of the first one.

\subsection{Skeleta and coskeleta}

For $n \geqslant 0$, let $\operatorname{sk}_{n}: \mathcal{S} \rightarrow \mathcal{S}$ be the $n$th skeleton functor for simplicial sets. It is the left adjoint of the $\operatorname{coskeleton}$ functor $\operatorname{cosk}_{n}: \mathcal{S} \rightarrow \mathcal{S}$. For a simplicial set $Z$, the simplicial set $\operatorname{sk}_{n} Z$ is given by the subspace of $Z$ generated by simplices of degree less than or equal to $n$. The $k$-simplices of the $n$th coskeleton $\operatorname{cosk}_{n} Z$ are given by the set $\operatorname{Hom}_{\mathcal{S}}\left(\operatorname{sk}_{n} \Delta[k], Z\right)$. If $Z=Y$ is a profinite space, then $\operatorname{cosk}_{n} Y$ inherits the structure of a profinite space. For $\operatorname{sk}_{n} \Delta[k]$ is a finite simplicial set, and hence the set

$$
\operatorname{Hom}_{\mathcal{S}}\left(\operatorname{sk}_{n} \Delta[k],|Y|\right) \cong \operatorname{Hom}_{\hat{\mathcal{S}}}\left(\operatorname{sk}_{n} \Delta[k], Y\right)
$$

inherits the structure as a profinite set.

Moreover, if $Y$ is a profinite $G$-space, then $\operatorname{Hom}_{\hat{\mathcal{S}}}\left(\operatorname{sk}_{n} \Delta[k], Y\right)$ is a limit of finite discrete $G$-sets, since $\operatorname{sk}_{n} \Delta[k]$ is a finite simplicial set with trivial $G$-action. Thus the well-known constructions for skeleta and coskeleta yield a pair of adjoint endofunctors $\left(\mathrm{sk}_{n}, \operatorname{cosk}_{n}\right)$ on $\hat{\mathcal{S}}_{G}$.

In terms of mapping spaces, this adjunction translates into the natural isomorphism of pointed simplicial sets

$$
\operatorname{Map}_{G}\left(\operatorname{sk}_{n} W, Y\right) \cong \operatorname{Map}_{G}\left(W, \operatorname{cosk}_{n} Y\right)
$$

for every profinite $G$-space $W$ and every pointed profinite $G$-space $Y$. The basepoint of $\operatorname{cosk}_{n} Y$ is given by the basepoint of $Y$.

Now let $X$ be an $\Omega$-spectrum in $\operatorname{Sp}\left(\hat{\mathcal{S}}_{* G}\right)$. For a given integer $n$, the $n$th coskeleton of $X$ is defined to be the profinite $G$-spectrum whose $k$ th space is the pointed profinite $G$-space $\operatorname{cosk}_{n+k} X_{k}$, i.e., the $(n+k)$ th coskeleton of the $k$ th space $X_{k}$ of $X$, where 
$\operatorname{cosk}_{n+k} X_{k}$ is defined to be the one point space if $n+k<0$. Each $\operatorname{cosk}_{n+k} X_{k}$ is a fibrant pointed profinite $G$-space and the induced map of pointed profinite $G$-spaces

$$
\operatorname{cosk}_{n+k} X_{k} \rightarrow \Omega\left(\operatorname{cosk}_{n+k+1} X_{k+1}\right)
$$

is a weak equivalence in $\hat{\mathcal{S}}_{* G}$, since $\operatorname{cosk}_{n}$ preserves fibrant objects and weak equivalences between fibrant objects. Thus $\operatorname{cosk}_{n} X$ is an $\Omega$-spectrum in $\operatorname{Sp}\left(\hat{\mathcal{S}}_{* G}\right)$ if $X$ is.

Lemma 3.13. Let $X$ be an $\Omega$-spectrum in $\operatorname{Sp}\left(\hat{\mathcal{S}}_{* G}\right)$. The tower

$$
\cdots \rightarrow \operatorname{cosk}_{2} X \rightarrow \operatorname{cosk}_{1} X \rightarrow \operatorname{cosk}_{0} X \rightarrow \operatorname{cosk}_{-1} X \rightarrow \cdots
$$

is a Postnikov tower for $X$, i.e., $X=\lim _{n} \operatorname{cosk}_{n} X, \pi_{q} X \rightarrow \pi_{q}\left(\operatorname{cosk}_{n} X\right)$ is an isomorphism if $q \leqslant n$, and $\pi_{q}\left(\cos _{n} X\right)=0$ if $q>n$. In particular, the fiber $F(n)$ of $\operatorname{cosk}_{n} X \rightarrow \operatorname{cosk}_{n-1} X$ is an Eilenberg-MacLane spectrum in $\operatorname{Sp}\left(\hat{\mathcal{S}}_{* G}\right)$ whose only nontrivial homotopy group is $\pi_{n} F(n) \cong \pi_{n} X$.

Proof. Let $n$ and $q$ be integers, and $k$ be any positive integer such that $q+k \geqslant 0$. Since $X$ and $\operatorname{cosk}_{n} X$ are $\Omega$-spectra in $\operatorname{Sp}\left(\hat{\mathcal{S}}_{* G}\right)$, the $q$ th profinite homotopy group of $X$ is given by the abelian profinite group $\pi_{q+k} X_{k}$, and the $q$ th homotopy group of $\operatorname{cosk}_{n} X$ is given by $\pi_{q+k}\left(\left(\operatorname{cosk}_{n} X\right)_{k}\right)=\pi_{q+k}\left(\operatorname{cosk}_{n+k} X_{k}\right)$. Hence $\pi_{q}\left(\operatorname{cosk}_{n} X\right)$ is isomorphic to $\pi_{q} X$ if $q+k \leqslant n+k$, i.e., if $q \leqslant n$, and $\pi_{q}\left(\operatorname{cosk}_{n} X\right)=0$ if $q+k>n+k$, i.e., if $q>n$.

Since $\operatorname{cosk}_{n} X$ and $\operatorname{cosk}_{n-1} X$ are $\Omega$-spectra in $\operatorname{Sp}\left(\hat{\mathcal{S}}_{* G}\right)$, the fiber $F(n)$ of the map $\operatorname{cosk}_{n} X \rightarrow \operatorname{cosk}_{n-1} X$ is an $\Omega$-spectrum in $\operatorname{Sp}\left(\hat{\mathcal{S}}_{* G}\right)$ by Proposition 3.12. For $k \geqslant 0$, the $k$ th space $F(n)_{k}$ is the fiber of $\operatorname{cosk}_{n+k} X_{k} \rightarrow \operatorname{cosk}_{n-1+k} X_{k}$. This fiber is the profinite $G$-space $K\left(\pi_{n+k} X_{k}, n+k\right)$. The structure map is given by the natural equivalence of pointed profinite $G$-spaces $K\left(\pi_{n+k} X_{k}, n+k\right) \rightarrow \Omega\left(K\left(\pi_{n+k} X_{k}, n+k+1\right)\right)$. Since $X$ is an $\Omega$-spectrum in $\operatorname{Sp}\left(\hat{\mathcal{S}}_{* G}\right)$, the profinite $G$-module $\pi_{n+k} X_{k}$ is the $n$th homotopy group of $X$. Hence the fiber $F(n)$ is an Eilenberg-MacLane spectrum whose homotopy groups $\pi_{m} F(n)$ vanish for $m \neq n$ and whose $n$th homotopy group is isomorphic to $\pi_{n} X$.

\subsection{Homotopy fixed point spectra}

As an example of a continuous mapping spectrum, let $E G$ be a contractible profinite $G$-space with a levelwise free $G$-action, in other words a cofibrant profinite $G$ space which is weakly equivalent to a point.

Definition 3.14. Let $X \in \operatorname{Sp}\left(\hat{\mathcal{S}}_{* G}\right)$ be a profinite $G$-spectrum and let $R_{G}$ be a fixed functorial fibrant replacement in $\operatorname{Sp}\left(\hat{\mathcal{S}}_{* G}\right)$. We define the homotopy fixed point spectrum $X^{h G}$ of $X$ to be the function spectrum of continuous $G$-equivariant maps from $E G$ to $R_{G} X$, i.e.,

$$
X^{h G}:=\operatorname{Map}_{G}\left(E G, R_{G} X\right) .
$$

Proposition 3.15. If $f: X \rightarrow Y$ is a stable equivalence of profinite $G$-spectra, then the induced map $f^{h G}: X^{h G} \rightarrow Y^{h G}$ is a stable equivalence between $\Omega$-spectra in $\operatorname{Sp}\left(\mathcal{S}_{*}\right)$.

Proof. If $f$ is a stable equivalence in $\operatorname{Sp}\left(\hat{\mathcal{S}}_{* G}\right)$, then $R_{G}(f)$ is a stable equivalence between $\Omega$-spectra in $\operatorname{Sp}\left(\hat{\mathcal{S}}_{* G}\right)$. Now the assertion follows from Lemma 3.10, Lemma 3.11, and the fact that $E G$ is cofibrant in $\hat{\mathcal{S}}_{G}$. 
Remark 3.16. 1. One should note that the homotopy fixed points of a profinite $G$ spectrum are not the homotopy limit of the $G$-action in the sense of the construction of the previous section. The homotopy limit construction would treat $G$ as an abstract group, or rather the category defined by $G$, and would forget the profinite topology. But for the homotopy fixed points we want to remember the topology of $G$. This is why we use the explicit functor $\operatorname{Map}_{G}(E G,-)$.

2. The reader may wonder why we do not look for homotopy fixed point spectra that are profinite spectra themselves. The goal of our construction is to obtain the descent spectral sequence of Theorem 3.17. Since the cohomology groups $H^{s}\left(G ; \pi_{t} X\right)$ are not profinite groups in general, there is no reason to expect $\pi_{*}\left(X^{h G}\right)$ to be profinite.

Before we prove our main result about continuous homotopy fixed point spectra, let us choose a concrete model for $E G$. We let $E G$ be the profinite $G$-space given in degree $n$ by the $(n+1)$-fold product $G^{n+1}$ of copies of $G$. Its $i$ th face map is given by

$$
d^{i}: G^{n+1} \rightarrow G^{n},\left(g_{1}, \ldots, g_{n+1}\right) \mapsto\left(g_{1}, \ldots, \hat{g_{i+1}}, \ldots, g_{n+1}\right)
$$

where the hat means that the component $g_{i+1}$ is omitted. We let $g \in G$ act on $G$ by $h \mapsto g h$, for all $h \in G$, and let $G$ act on $E G_{n}$ via the diagonal action on $G^{n+1}$. This action is free, and the quotient $E G / G$ is the usual classifying space $B G$ for $G$ given in degree $n$ by $G^{n}$ with face maps

$$
\bar{d}^{i}\left(g_{1}, \ldots, g_{n}\right)= \begin{cases}\left(g_{2}, \ldots, g_{n}\right) & : i=0 \\ \left(g_{1}, \ldots, g_{i} g_{i+1}, \ldots, g_{n}\right) & : \quad 1 \leqslant i \leqslant n-1 \\ \left(g_{1}, \ldots, g_{n-1}\right) & : i=n .\end{cases}
$$

Theorem 3.17. Let $G$ be a profinite group and $X$ a profinite $G$-spectrum. There is a homotopy fixed point spectral sequence whose $E_{2}^{s, t}$-term is the sth continuous cohomology of $G$ with coefficients the profinite $G$-module $\pi_{t} X$ :

$$
E_{2}^{s, t}=H^{s}\left(G ; \pi_{t} X\right) \Rightarrow \pi_{t-s}\left(X^{h G}\right) .
$$

This spectral sequence converges completely to $\pi_{*}\left(X^{h G}\right)$ if $\lim _{r}^{1} E_{r}^{s, t}=0$ for all $s, t$.

The reader should note that above and in the rest of the paper we do not use a special notation for continuous cohomology. For a profinite space and a topological coefficient group, cohomology will always mean continuous cohomology.

Proof. After applying the fibrant replacement functor $R_{G}$ we can assume that $X$ is fibrant in $\operatorname{Sp}\left(\hat{\mathcal{S}}_{* G}\right)$. Let $\operatorname{sk}_{n} E G$ be the $n$th skeleton of $E G$ in $\hat{\mathcal{S}}_{G}$. The induced $G$ equivariant map sk ${ }_{n-1} E G \rightarrow \mathrm{sk}_{n} E G$ is a cofibration of profinite $G$-spaces (since we only add free copies of $G$ in dimension $n$ as nondegenerate simplices). Filtering $E G$ by its finite skeleta we obtain a tower of spectra

$$
\begin{gathered}
\cdots \rightarrow \operatorname{Map}_{G}\left(\operatorname{sk}_{n} E G, X\right) \rightarrow \operatorname{Map}_{G}\left(\operatorname{sk}_{n-1} E G, X\right) \rightarrow \cdots \\
\left\{X(n):=\operatorname{Map}_{G}\left(\operatorname{sk}_{n} E G, X\right)\right\}_{n}
\end{gathered}
$$

whose limit is isomorphic to $X^{h G}$. Since the skeleton $\operatorname{sk}_{n} E G$ is a cofibrant profinite $G$ space for every $n \geqslant 0$, every spectrum $\operatorname{Map}_{G}\left(\operatorname{sk}_{n} E G, X\right)$ is an $\Omega$-spectrum in $\operatorname{Sp}\left(\mathcal{S}_{*}\right)$ 
by Lemma 3.10, and each map

$$
\operatorname{Map}_{G}\left(\operatorname{sk}_{n} E G, X\right) \rightarrow \operatorname{Map}_{G}\left(\operatorname{sk}_{n-1} E G, X\right)
$$

is a projective (or, in the terminology used in [2], a strict) fibration in $\operatorname{Sp}\left(\mathcal{S}_{*}\right)$ by Lemma 3.9. Now we can use the fact that the projective fibrations between $\Omega$-spectra are exactly the stable fibrations. For $\operatorname{Sp}\left(\mathcal{S}_{*}\right)$ this was proved in [2], Lemma A.8. But this is just a special case of the general fact that in a left Bousfield localization $L_{\mathcal{C}} \mathcal{M}$ of a model category $\mathcal{M}$ a map between $\mathcal{C}$-local objects is a fibration in $L_{\mathcal{C}} \mathcal{M}$ if and only if it is a fibration in $\mathcal{M}$ by $[\mathbf{1 3}]$. Thus (8) is in fact a tower of stable fibrations between $\Omega$-spectra in $\operatorname{Sp}\left(\mathcal{S}_{*}\right)$.

By the adjunction of skeleta and coskeleta, tower (8) is naturally isomorphic to the tower of stable fibrations of $\Omega$-spectra in $\operatorname{Sp}\left(\mathcal{S}_{*}\right)$

$$
\cdots \rightarrow \operatorname{Map}_{G}\left(E G, \operatorname{cosk}_{n} X\right) \rightarrow \operatorname{Map}_{G}\left(E G, \operatorname{cosk}_{n-1} X\right) \rightarrow \cdots
$$

By Lemma 3.13, the fiber of the map $\operatorname{cosk}_{n} X \rightarrow \operatorname{cosk}_{n-1} X$ is an Eilenberg-MacLane spectrum $H \pi_{n} X[n]$. Since $\pi_{n} X$ is a profinite $G$-module, $H \pi_{n} X[n]$ is a fibrant object of $\operatorname{Sp}\left(\hat{\mathcal{S}}_{* G}\right)$ whose $k$ th space is the pointed profinite $G$-space $K\left(\pi_{n} X, k+n\right)$. Now the crucial point is that the functor $\operatorname{Map}_{G}(E G,-)$ sending $\Omega$-spectra in $\operatorname{Sp}\left(\hat{\mathcal{S}}_{* G}\right)$ to $\Omega$-spectra in $\operatorname{Sp}\left(\mathcal{S}_{*}\right)$ preserves homotopy fibers by Proposition 3.12. Hence, for every $n$, tower (9) induces a long exact sequence of abelian groups

$$
\begin{aligned}
\cdots \rightarrow \pi_{k+1}\left(\operatorname{Map}_{G}\left(E G, \operatorname{cosk}_{n-1} X\right)\right) & \rightarrow \pi_{k}\left(\operatorname{Map}_{G}(E G, F(n))\right) \rightarrow \\
& \rightarrow \pi_{k}\left(\operatorname{Map}_{G}\left(E G, \operatorname{cosk}_{n} X\right)\right) \rightarrow \cdots
\end{aligned}
$$

continuing with $\pi_{k}\left(\operatorname{Map}_{G}\left(E G, \operatorname{cosk}_{n-1} X\right)\right)$ and so on. This sequence yields exact couples for various $n$ and we obtain a spectral sequence

$$
E_{2}^{s, t}=\pi_{t-s}\left(\operatorname{Map}_{G}(E G, F(t))\right) \Rightarrow \pi_{t-s}\left(X^{h G}\right) .
$$

Since $F(t)$ is an $\Omega$-spectrum in $\operatorname{Sp}\left(\hat{\mathcal{S}}_{* G}\right)$ equivalent to $H \pi_{t} X[t]$, the fiber of the map

$$
\operatorname{Map}_{G}\left(E G, \operatorname{cosk}_{t} X\right) \rightarrow \operatorname{Map}_{G}\left(E G, \operatorname{cosk}_{t-1} X\right)
$$

is the $\Omega$-spectrum $\operatorname{Map}_{G}\left(E G, H \pi_{t} X[t]\right)$ in $\operatorname{Sp}\left(\mathcal{S}_{*}\right)$. The $(t-s)$ th homotopy group of this spectrum is given by any of the isomorphic abelian groups

$$
\pi_{t-s+k}\left(\operatorname{Map}_{G}\left(E G, K\left(\pi_{t+k} X_{k}, t+k\right)\right)\right)
$$

such that $t+k \geqslant 0$. The following lemma shows that this group is exactly the continuous cohomology group $H^{s}\left(G ; \pi_{t} X\right)$. Finally, complete convergence follows as in $[\mathbf{3}]$, IX $\S 5$.

To finish the proof of Theorem 3.17, it remains to prove the following lemma.

Lemma 3.18. Let $\pi$ be a profinite $G$-module. For every $n \geqslant 0$ and $0 \leqslant q \leqslant n$, there is a natural isomorphism

$$
\pi_{q}\left(\operatorname{Map}_{G}(E G, K(\pi, n))\right) \cong H^{n-q}(G ; \pi)
$$

between the homotopy groups of the fibrant pointed space $\operatorname{Map}_{G}(E G, K(\pi, n))$ and the continuous cohomology of $G$ with coefficients in $\pi$. Moreover, for $q>n$, the group $\pi_{q}\left(\operatorname{Map}_{G}(E G, K(\pi, n))\right)$ vanishes. 
Proof. The weak equivalence $K(\pi, n) \rightarrow \Omega(K(\pi, n+1))$ of pointed profinite $G$-spaces induces a weak equivalence

$$
\operatorname{Map}_{G}(E G, K(\pi, n)) \rightarrow \Omega\left(\operatorname{Map}_{G}(E G, K(\pi, n+1))\right)
$$

of pointed simplicial sets. Hence by induction it suffices to show

$$
\pi_{0}\left(\operatorname{Map}_{G}(E G, K(\pi, n))\right) \cong H^{n}(G ; \pi),
$$

since $\Omega^{i} K(\pi, 0)$ is contractible for every $i \geqslant 1$. For a profinite $G$-space $Y$ recall the cochain complex $C^{*}(Y ; \pi)$ given in degree $n$ by the set of continuous cocycles in the cochain complex of continuous maps

$$
C^{n}(Y ; \pi)=\operatorname{Hom}_{\hat{\mathcal{E}}}\left(Y_{n}, \pi\right) .
$$

The differential $\delta: C^{n}(Y ; \pi) \rightarrow C^{n+1}(Y ; \pi)$ is the map associating to the continuous map $\alpha: Y_{n} \rightarrow \pi$ the map $\sum_{i=0}^{n+1}(-1)^{i} \alpha \circ d_{i}$, where $d_{i}$ denotes the $i$ th face map of $Y$. For $Y=E G, C^{n}(E G ; \pi)$ is just the set

$$
C^{n}(G ; \pi)=\operatorname{Hom}_{\hat{\mathcal{E}}}\left(G^{n+1}, \pi\right)
$$

of continuous maps from the $(n+1)$-fold product of copies of $G$ to $\pi$ and $\delta^{n}$ sends a map $\alpha: G^{n+1} \rightarrow \pi$ to the map given by

$$
\left(g_{0}, \ldots, g_{n+1}\right) \mapsto \sum_{i=0}^{n+1}(-1)^{i} \alpha\left(g_{0}, \ldots, \hat{g}_{i}, \ldots, g_{n+1}\right) .
$$

The subcomplex of $G$-equivariant continuous maps $C_{G}^{*}(G ; \pi) \subset C^{*}(G ; \pi)$ is given in degree $n$ by the maps $\alpha$ such that

$$
g \alpha\left(g_{1}, \ldots, g_{n+1}\right)=\alpha\left(g g_{1}, \ldots, g g_{n+1}\right) .
$$

The homology groups of this cochain complex are the continuous cohomology groups of $G$ with coefficients in the profinite $G$-module $\pi$. Since the differentials in $C^{*}(G ; \pi)$ are $G$-equivariant, the group of $n$th cocycles $Z_{G}^{n}(G ; \pi)$ in the complex $C_{G}^{*}(G ; \pi)$ is equal to the subgroup of $G$-equivariant maps in $Z^{n}(G ; \pi)$. Hence in order to prove the lemma it suffices to show that there is an isomorphism

$$
\pi_{0}\left(\operatorname{Map}_{G}(E G, K(\pi, n))\right) \cong H_{G}^{n}(E G ; \pi)
$$

where $H_{G}^{n}(E G ; \pi)$ denotes the $n$th cohomology group of the complex $C_{G}^{*}(E G ; \pi)$ of $G$-equivariant cochains. Since $E G$ is cofibrant and $K(\pi, n)$ is a fibrant profinite $G$ space, there is a canonical bijection between the set $\pi_{0}\left(\operatorname{Map}_{G}(E G, K(\pi, n))\right)$ and the set of homotopy classes of maps in $\hat{\mathcal{S}}_{G}$ from $E G$ to $K(\pi, n)$, i.e., the set of equivalence classes of $\operatorname{Hom}_{\hat{\mathcal{S}}_{G}}(E G, K(\pi, n))$ modulo simplicial homotopy. Hence it suffices to show that, for any cofibrant profinite $G$-space $Y$, there is an isomorphism

$$
\phi: \operatorname{Hom}_{\hat{\mathcal{S}}_{G}}(Y, K(\pi, n)) / \sim \stackrel{\cong}{\rightrightarrows} H_{G}^{n}(Y ; \pi),
$$

where $\sim$ denotes the equivalence relation generated by simplicial homotopy.

The rest of the argument is almost exactly the same as in [20], 24 , we just have to take into account that our maps and cocycles are $G$-equivariant. For the convenience of the reader we repeat the main ideas of $[\mathbf{2 0}], \S 24$, for which we do not claim any originality. 
The crucial point is that the fibrant profinite $G$-space $K(\pi, n)$ represents $G$ equivariant continuous cohomology in $\hat{\mathcal{S}}_{G}$ with coefficients in the profinite abelian $G$ module $\pi$. To see how this works, recall the simplicial profinite $G$-module $L(\pi, n+1)$ given in simplicial degree $q$ by the group $C^{n}(\Delta[q] ; \pi)$ with $G$-action induced by the one on $\pi$. Define an $n$-cochain $u \in C^{n}(L(\pi, n+1) ; \pi)$ by $u(\alpha)=\alpha\left(\operatorname{id}_{[n]}\right)$ where $\alpha \in C^{n}(\Delta[n], \pi)$. It follows immediately from its definition that $u$ is $G$-equivariant, i.e., that it lies in the subgroup $C_{G}^{n}(L(\pi, n+1) ; \pi)$.

Let $Y$ be a cofibrant profinite $G$-space. The abelian group structure on $\pi$ induces a group structure on $\operatorname{Hom}_{\hat{\mathcal{S}}_{G}}(Y, L(\pi, n+1))$. Define a homomorphism

$$
\phi: \operatorname{Hom}_{\hat{\mathcal{S}}_{G}}(Y, L(\pi, n+1)) \cong C_{G}^{n}(Y ; \pi)
$$

by $\phi(f):=f^{*}(u)$ where $f^{*}$ is the pullback induced by $f$. We note that, for a $G$ equivariant $f, f^{*}(u)$ is also $G$-equivariant. One can show exactly as in $[\mathbf{2 0}]$, Lemma 24.2 , that $\phi$ is an isomorphism whose inverse

$$
\psi: C_{G}^{n}(Y ; \pi) \rightarrow \operatorname{Hom}_{\hat{\mathcal{S}}_{G}}(Y, L(\pi, n+1))
$$

is given by sending $\gamma \in C_{G}^{n}(Y ; \pi)$ to the map $\psi(\gamma)$ defined by $\psi(\gamma)(x)=\bar{x}^{*}(\gamma)$ for $x \in Y_{q}$, which we consider as a simplicial map $\Delta[q] \rightarrow Y$ that induces a homomorphism $\bar{x}^{*}: C_{G}^{n}(Y ; \pi) \rightarrow C^{n}(\Delta[q] ; \pi)$. The map $\psi(\gamma)$ is in fact $G$-equivariant. For the simplicial map $\psi(\gamma)$ is defined in degree $q$ by sending $x$ to the element in $L(\pi, n+1)_{q}$ given by the composite

$$
\Delta[q] \stackrel{x}{\rightarrow} Y_{n} \stackrel{\gamma}{\rightarrow} \pi
$$

Now for $g \in G, \psi(\gamma)$ sends the element $g x \in Y_{q}$ to the element in $L(\pi, n+1)_{q}$ given by the composite

$$
\Delta[q] \stackrel{x}{\rightarrow} Y_{n} \stackrel{g}{\rightarrow} Y_{n} \stackrel{\gamma}{\rightarrow} \pi .
$$

But since $\gamma$ is $G$-equivariant by assumption, this map is the same as

$$
\Delta[q] \stackrel{x}{\rightarrow} Y_{n} \stackrel{\stackrel{\gamma}{\rightarrow}}{\rightarrow} \stackrel{g}{\rightarrow} \pi
$$

which is exactly $g \psi(\gamma)(x)$. Hence we get $\psi(\gamma)(g x)=g \psi(\gamma)(x)$.

Now the complex $K(\pi, n)$ is given in degree $q$ as the subgroup

$$
K(\pi, n)_{q}=Z^{n}(\Delta[q] ; \pi)
$$

of cocycles in $L(\pi, n+1)_{q}$. One checks as in [20], Lemma 24.3, that $u$ induces a fundamental cocycle in $Z_{G}^{n}(K(\pi, n), n)$ which is also denoted by $u$ and that $\phi$ induces an isomorphism

$$
\phi: \operatorname{Hom}_{\hat{\mathcal{S}}_{G}}(Y, K(\pi, n)) \cong Z_{G}^{n}(Y ; \pi)
$$

whose inverse is induced by the restriction of $\psi$. Hence it remains to show that under the isomorphism $\phi$ in (10) two maps

$$
f, f^{\prime} \in \operatorname{Hom}_{\hat{\mathcal{S}}_{G}}(Y, K(\pi, n))
$$

are homotopic if and only if $\phi(f)$ is cohomologous to $\phi\left(f^{\prime}\right)$ in $Z_{G}^{n}(Y ; \pi)$. This can be shown as in [20], Theorem 24.4. If $f$ and $f^{\prime}$ are homotopic, then it follows from the invariance of cohomology under homotopy that $f^{*}(u)$ and $f^{\prime *}(u)$ agree on the cohomology level and hence are cohomologous. Now suppose $\phi(f)=\phi\left(f^{\prime}\right)+\delta(\alpha)$, 
where $\delta$ is the boundary map and $\alpha$ is a cochain in $C_{G}^{n-1}(Y ; \pi)$. Let $i_{0}: Y \rightarrow Y \times 0$ and $i_{1}: Y \rightarrow Y \times 1$ be the simplicial maps identifying $Y$ with the cited subcomplexes of $Y \times \Delta[1]$, where we regard $\Delta[1]$ as before as a simplicial finite set with trivial $G$-action and where 0 and 1 denote the corresponding vertices $\Delta[0] \rightarrow \Delta[1]$ of $\Delta[1]$. It suffices to find a $\gamma \in Z_{G}^{n}(Y \times \Delta[1] ; \pi)$ such that

$$
i_{0}^{*}(\gamma)=\gamma_{\mid C_{G}^{n}(Y \times 0 ; \pi)}=\phi(f)
$$

and

$$
i_{1}^{*}(\gamma)=\gamma_{\mid C_{G}^{n}(Y \times 1 ; \pi)}=\phi\left(f^{\prime}\right) .
$$

For then the map $\psi(\gamma): Y \times \Delta[1] \rightarrow K(\pi, n)$ is a homotopy between $f$ and $f^{\prime}$. This homotopy is even $G$-equivariant, since $\psi(\gamma)$ is $G$-equivariant as shown above. Let $p: Y \times \Delta[1] \rightarrow Y$ be the projection onto $Y$ and let

$$
\gamma_{0}=p^{*}(\phi(f)) \in Z_{G}^{n}(Y \times \Delta[1] ; \pi) .
$$

Since $p \circ i_{0}=p \circ i_{1}=\mathrm{id}$, we have $i_{0}^{*}\left(\gamma_{0}\right)=i_{1}^{*}\left(\gamma_{0}\right)=\phi(f)$. Further, regarding $\alpha$ as a cochain defined on $i_{1}(Y)$, we may choose a cochain $\beta \in C_{G}^{n-1}(Y \times \Delta[1] ; \pi)$ which extends $\alpha$ and vanishes on $i_{0}(Y)$. Thus $i_{0}^{*}(\beta)=0$ and $i_{1}^{*}(\beta)=\alpha$. Now set

$$
\gamma:=\gamma_{0}-\delta(\beta)
$$

Then $i_{0}^{*}(\gamma)=\phi(f)$ and $i_{1}^{*}(\gamma)=\phi(f)-\delta(\alpha)=\phi\left(f^{\prime}\right)$, as desired.

3.4. A cosimplicial version of the homotopy fixed point spectral sequence

The homotopy fixed point spectral sequence of Theorem 3.17 and the definition of homotopy fixed points can also be given in terms of cosimplicial spectra and total spaces of cosimplicial objects. Let $V^{\bullet}$ be a cosimplicial pointed space, i.e., a cosimplicial object in $\mathcal{S}_{*}$. The total space $\operatorname{Tot} V^{\bullet}$ of $V^{\bullet}$ (see [3], X, §3) is given by the equalizer in $\mathcal{S}_{*}$ of the diagram

$$
\prod_{n \geqslant 0} \operatorname{hom}_{\mathcal{S}_{*}}\left(\Delta[n], V^{n}\right) \rightrightarrows \prod_{\varphi:[n] \rightarrow[m]} \operatorname{hom}_{\mathcal{S}_{*}}\left(\Delta[n], V^{m}\right) .
$$

For $k \geqslant 0$, let $\operatorname{Tot}_{k} V^{\bullet}$ be the equalizer in $\mathcal{S}_{*}$ of the diagram

$$
\prod_{n \geqslant 0} \operatorname{hom}_{\mathcal{S}_{*}}\left(\operatorname{sk}_{k} \Delta[n], V^{n}\right) \rightrightarrows \prod_{\varphi:[n] \rightarrow[m]} \operatorname{hom}_{\mathcal{S}_{*}}\left(\operatorname{sk}_{k} \Delta[n], V^{m}\right)
$$

Now let $Z^{\bullet}$ be a cosimplicial spectrum, i.e., a cosimplicial object in $\operatorname{Sp}\left(\mathcal{S}_{*}\right)$. The total spectrum $\operatorname{Tot} Z^{\bullet}$ is the spectrum whose $n$th space is the total space of the cosimplicial space $Z_{n}^{\bullet}$ (see [30], Definition 5.24). Since Tot commutes with cotensor objects for spaces, the structure maps of $\operatorname{Tot} Z^{\bullet}$ are given by the maps

$$
\operatorname{Tot} Z_{n}^{\bullet} \rightarrow \operatorname{Tot}\left(\Omega Z_{n+1}^{\bullet}\right) \cong \Omega\left(\operatorname{Tot} Z_{n+1}^{\bullet}\right) \text {. }
$$

Since cotensor objects in $\operatorname{Sp}\left(\mathcal{S}_{*}\right)$ are defined levelwise, Tot also commutes with cotensor objects for spectra. For $k \geqslant 0, \operatorname{Tot}_{k} Z \bullet$ is defined to be the spectrum obtained by applying $\operatorname{Tot}_{k}$ levelwise.

Now let $W$ be a profinite $G$-space and $X$ a profinite $G$-spectrum. Considering the $n$th profinite set $W_{n}$ of $W$ as a constant simplicial profinite $G$-space, we can view $W$ also as a simplicial object in $\hat{\mathcal{S}}_{G}$. Applying the functor $\operatorname{Map}_{G}(-, X)$ to $W$ yields a cosimplicial spectrum which we denote by $\operatorname{Map}_{G}\left(W^{\bullet}, X\right)$. 
Lemma 3.19. (a) For $W$ and $X$ as above, the total spectrum $\operatorname{Tot}\left(\operatorname{Map}_{G}\left(W^{\bullet}, X\right)\right)$ is naturally isomorphic to the spectrum $\operatorname{Map}_{G}(W, X)$.

(b) For every $k \geqslant 0$, the spectrum $\operatorname{Tot}_{k}\left(\operatorname{Map}_{G}\left(W^{\bullet}, X\right)\right)$ is naturally isomorphic to the spectrum $\operatorname{Map}_{G}\left(\operatorname{sk}_{k} W, X\right)$.

Proof. Since the total spectrum is defined levelwise, it suffices to prove the corresponding assertions when $X$ is a pointed profinite $G$-space. In this case, (a) follows immediately from the description of $\operatorname{Tot}\left(\operatorname{Map}_{G}\left(W^{\bullet}, X\right)\right)$ as the equalizer in $\mathcal{S}_{*}$ of the diagram

$$
\prod_{n \geqslant 0} \operatorname{hom}_{\mathcal{S}_{*}}\left(\Delta[n], \operatorname{Map}_{G}\left(W^{n}, X\right)\right) \rightrightarrows \prod_{\varphi:[n] \rightarrow[m]} \operatorname{hom}_{\mathcal{S}_{*}}\left(\Delta[n], \operatorname{Map}_{G}\left(W^{m}, X\right)\right) .
$$

Claim (b) follows in the same way by considering the corresponding equalizer diagram for $\operatorname{Tot}_{k}$.

For $W=E G \in \hat{\mathcal{S}}_{G}$, we obtain a cosimplicial spectrum $\operatorname{Map}_{G}\left(G^{\bullet+1}, X\right)$ whose $n$th spectrum is $\operatorname{Map}_{G}\left(G^{n+1}, X\right)$ (different versions of this object have already been studied in [30]). The previous lemma and the construction of the homotopy fixed point spectral sequence in the proof of Theorem 3.17 imply the following result.

Proposition 3.20. Let $X$ be a fibrant profinite $G$-spectrum.

(a) There is a natural isomorphism of spectra

$$
X^{h G}=\operatorname{Map}_{G}(E G, X) \cong \operatorname{Tot}\left(\operatorname{Map}_{G}\left(G^{\bullet+1}, X\right)\right) .
$$

(b) The spectral sequence of Theorem 3.17 is isomorphic to the spectral sequence associated to the tower of spectra

$$
\left\{\operatorname{Tot}_{k}\left(\operatorname{Map}_{G}\left(G^{\bullet+1}, X\right)\right)\right\}_{k} .
$$

In [30], Definition 5.23, Thomason calls a cosimplicial spectrum $Z^{\bullet}$ a cosimplicial fibrant spectrum, if each spectrum $Z^{n}$ is a fibrant spectrum, and calls $Z^{\bullet}$ a fibrant cosimplicial fibrant spectrum if in addition each cosimplicial space $Z_{m}^{\bullet}$ is Reedy fibrant (see [13], 15.3.2, or [3], X, 4.6).

Lemma 3.21. (a) Let $V$ be a fibrant pointed profinite $G$-space. Then $\operatorname{Map}_{G}\left(G^{\bullet+1}, V\right)$ is a fibrant cosimplicial space in the Reedy model category structure on cosimplicial spaces.

(b) Let $X$ be a fibrant profinite $G$-spectrum. Then $\operatorname{Map}_{G}\left(G^{\bullet+1}, X\right)$ is a fibrant cosimplicial fibrant spectrum.

Proof. (a) By [13], Lemma 15.11.10, in order to show that $\operatorname{Map}_{G}\left(G^{\bullet+1}, V\right)$ is Reedy fibrant it suffices to show that $G^{\bullet+1}$ is a Reedy cofibrant simplicial profinite $G$-space. This means that the map from the $n$th latching object $L_{n} G^{\bullet+1}$ to $G^{n+1}$ is a cofibration in $\hat{\mathcal{S}}_{G}$ for every $n \geqslant 0$. Since $L_{n} G^{\bullet+1}$ is a subobject of $G^{n+1}$ in $\hat{\mathcal{S}}$ and since the action of $G$ on $G^{n+1}$ is free, the map $L_{n} G^{\bullet+1} \rightarrow G^{n+1}$ is in fact a cofibration in $\hat{\mathcal{S}}_{G}$.

Claim (b) follows from (a) and the fact that $\operatorname{Map}_{G}\left(G^{n+1}, X\right)$ is an $\Omega$-spectrum for every $n$.

Finally, we would like to replace $\operatorname{Map}_{G}\left(G^{\bullet+1}, X\right)$ by a cosimplicial spectrum of the form $\operatorname{Map}\left(G^{\bullet}, X\right)$ given in cosimplicial degree $n$ by the spectrum $\operatorname{Map}\left(G^{n}, X\right)$. 
Remark 3.22. In the following discussion we abuse notation and describe elements in $\operatorname{Map}_{G}\left(G^{n+1}, X\right)$ or $\operatorname{Map}\left(G^{n}, X\right)$ simply by their effects on tuples of elements in $G$ and neglect that a general element in the $k$ th set $\operatorname{Map}_{G}\left(G^{n+1}, X_{m}\right)_{k}$ of the $m$ th space is a $G$-equivariant map $G^{n+1} \times \Delta[k] \rightarrow X_{m}$, respectively a map $G^{n} \times \Delta[k] \rightarrow X_{m}$ in $\operatorname{Map}\left(G^{n}, X_{m}\right)_{k}$. Moreover, we will only discuss the coface maps, since they are important for the resulting cochain structures, and omit the calculations for the codegeneracy maps.

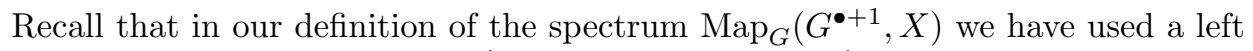
$G$-action such that a map $\beta: G^{n+1} \rightarrow X$ is in $\operatorname{Map}_{G}\left(G^{n+1}, X\right)$ if it satisfies

$$
\alpha\left(g_{1}, \ldots, g_{n+1}\right)=g \alpha\left(g^{-1} g_{1}, \ldots, g^{-1} g_{n+1}\right)
$$

for every $g \in G$.

The $i$ th coface map

$$
d^{i}: \operatorname{Map}_{G}\left(G^{n}, X\right) \rightarrow \operatorname{Map}_{G}\left(G^{n+1}, X\right)
$$

is given by sending a map $\alpha: G^{n} \rightarrow X$ to $d^{i}(\alpha): G^{n+1} \rightarrow X$ that sends the $(n+1)$ tuple $\left(g_{1}, \ldots, g_{n+1}\right)$ to $\alpha\left(g_{1}, \ldots, g_{i+1}, \ldots, g_{n+1}\right)$.

Now for the cosimplicial object $\operatorname{Map}\left(G^{\bullet}, X\right)$ we define the $i$ th coface map

$$
\tilde{d}^{i}: \operatorname{Map}\left(G^{n-1}, X\right) \rightarrow \operatorname{Map}\left(G^{n}, X\right)
$$

to be the map which sends a map $\beta: G^{n-1} \rightarrow X$ to the map

$$
\tilde{d}^{i}(\beta)\left(g_{1}, \ldots, g_{n}\right)= \begin{cases}g_{1} \beta\left(g_{2}, \ldots, g_{n}\right) & : i=0 \\ \beta\left(g_{1}, \ldots, g_{i} g_{i+1}, \ldots, g_{n}\right) & : \quad 1 \leqslant i \leqslant n-1 \\ \beta\left(g_{1}, \ldots, g_{n-1}\right) & : \quad i=n .\end{cases}
$$

We then have a morphism $\varphi: \operatorname{Map}_{G}\left(G^{\bullet+1}, X\right) \rightarrow \operatorname{Map}\left(G^{\bullet}, X\right)$ of cosimplicial spectra defined as follows. For $n=0$, we have

$$
\operatorname{Map}_{G}(G, X) \rightarrow \operatorname{Map}(*, X)=X, \alpha \mapsto \alpha(1) .
$$

For $n \geqslant 1$,

$$
\operatorname{Map}_{G}\left(G^{n+1}, X\right) \rightarrow \operatorname{Map}\left(G^{n}, X\right), \alpha \mapsto \varphi(\alpha)=\beta
$$

where $\beta$ is defined by

$$
\beta\left(g_{1}, \ldots, g_{n}\right):=\alpha\left(1, g_{1}, g_{1} g_{2}, g_{1} g_{2} g_{3}, \ldots, g_{1} \cdots g_{n}\right) .
$$

The inverse $\varphi^{-1}$ is given by the map that sends $\beta \in \operatorname{Map}\left(G^{n}, X\right)$ to the element $\alpha=\varphi^{-1}(\beta) \in \operatorname{Map}_{G}\left(G^{n+1}, X\right)$ defined by

$$
\alpha\left(g_{0}, \ldots, g_{n}\right):=g_{0} \beta\left(g_{0}^{-1} g_{1}, g_{1}^{-1} g_{2}, \ldots, g_{n-1}^{-1} g_{n}\right) .
$$

In the following we assume $n \geqslant 1$, and leave the case $n=0$ for the reader. The map $\varphi^{-1}(\beta)$ is in fact $G$-equivariant. For we have for every $g \in G$

$$
\begin{aligned}
g \varphi^{-1}(\beta)\left(g_{0}, \ldots, g_{n}\right) & =g g_{0} \beta\left(g_{0}^{-1} g_{1}, g_{1}^{-1} g_{2}, \ldots, g_{n-1}^{-1} g_{n}\right) \\
& =g g_{0} \beta\left(\left(g g_{0}\right)^{-1} g g_{1}, \ldots,\left(g g_{n-1}\right)^{-1} g g_{n}\right) \\
& =\varphi^{-1}(\beta)\left(g g_{0}, \ldots, g g_{n}\right) .
\end{aligned}
$$


Now one can check

$$
\begin{aligned}
\varphi^{-1}(\varphi(\alpha))\left(g_{1}, \ldots, g_{n}\right) & =g_{1} \varphi(\alpha)\left(g_{1}^{-1} g_{2}, \ldots, g_{n-1}^{-1} g_{n}\right) \\
& =g_{1} \alpha\left(1, g_{1}^{-1} g_{2}, g_{1}^{-1} g_{2} g_{1}^{-1} g_{3}, \ldots, g_{1}^{-1} g_{2} \cdots g_{n-1}^{-1} g_{n}\right) \\
& =\alpha\left(g_{1}, \ldots, g_{n}\right) .
\end{aligned}
$$

On the other hand we have

$$
\begin{aligned}
\varphi\left(\varphi^{-1}(\beta)\right)\left(g_{1}, \ldots, g_{n-1}\right) & =\varphi^{-1}(\beta)\left(1, g_{1}, g_{1} g_{2}, \ldots, g_{1} \cdots g_{n-1}\right) \\
& =\beta\left(g_{1}, g_{1}^{-1} g_{1} g_{2}, \ldots,\left(g_{1} \cdots g_{n-2}\right)^{-1} g_{1} \cdots g_{n-1}\right) \\
& =\beta\left(g_{1}, \ldots, g_{n-1}\right) .
\end{aligned}
$$

Moreover, the maps $\varphi$ and $\varphi^{-1}$ are compatible with the coface maps. We start with $\varphi$.

For $i=0$, we have

$$
\begin{aligned}
\varphi\left(d^{0}(\alpha)\right)\left(g_{1}, \ldots, g_{n}\right) & =d^{0}(\alpha)\left(1, g_{1}, g_{1} g_{2}, \ldots, g_{1} \cdots g_{n}\right) \\
& =\alpha\left(\hat{1}, g_{1}, g_{1} g_{2}, \ldots, g_{1} \cdots g_{n}\right) \\
& =\alpha\left(g_{1}, g_{1} g_{2}, \ldots, g_{1} \cdots g_{n}\right) \\
& =g_{1} \varphi(\alpha)\left(g_{2}, \ldots, g_{n}\right) \\
& =\tilde{d}^{0}(\varphi(\alpha))\left(g_{1}, \ldots, g_{n}\right) .
\end{aligned}
$$

For $1 \leqslant i \leqslant n-1$, we have

$$
\begin{aligned}
\varphi\left(d^{i}(\alpha)\right)\left(g_{1}, \ldots, g_{n}\right) & =d^{i}(\alpha)\left(1, g_{1}, g_{1} g_{2}, \ldots, g_{1} \cdots g_{n}\right) \\
& =\alpha\left(1, g_{1}, g_{1} g_{2}, \ldots, g_{1} \cdots g_{i}, \ldots, g_{1} \cdots g_{n}\right) \\
& =\varphi(\alpha)\left(g_{1}, \ldots, g_{i} g_{i+1}, \ldots, g_{n}\right) \\
& =\tilde{d}^{i}(\varphi(\alpha))\left(g_{1}, \ldots, g_{n}\right) .
\end{aligned}
$$

For $i=n$, we have

$$
\begin{aligned}
\varphi\left(d^{n}(\alpha)\right)\left(g_{1}, \ldots, g_{n}\right) & =d^{n}(\alpha)\left(1, g_{1}, g_{1} g_{2}, \ldots, g_{1} \cdots g_{n}\right) \\
& =\alpha\left(1, g_{1}, \ldots, g_{1} \cdots g_{n-1}\right) \\
& =\varphi(\alpha)\left(g_{1}, \ldots, g_{n-1}\right) \\
& =\tilde{d^{n}}(\varphi(\alpha))\left(g_{1}, \ldots, g_{n}\right) .
\end{aligned}
$$

Now we check $\varphi^{-1}$. For $i=0$, we have

$$
\begin{aligned}
\varphi^{-1}\left(\tilde{d}^{0}(\beta)\right)\left(g_{0}, \ldots, g_{n}\right) & =g_{0} \tilde{d}^{0}(\beta)\left(g_{0}^{-1} g_{1}, g_{1}^{-1} g_{2}, \ldots, g_{n-1}^{-1}\right) \\
& =g_{0} g_{0}^{-1} g_{1} \beta\left(g_{1}^{-1} g_{2}, \ldots, g_{n-1}^{-1} g_{n}\right) \\
& =g_{1} \beta\left(g_{1}^{-1} g_{2}, \ldots, g_{n-1}^{-1} g_{n}\right) \\
& =\varphi^{-1}(\beta)\left(g_{1}, \ldots, g_{n}\right) \\
& =d^{0}\left(\varphi^{-1}(\beta)\right)\left(g_{0}, g_{1}, \ldots, g_{n}\right) .
\end{aligned}
$$

For $1 \leqslant i \leqslant n-1$, we have

$$
\begin{aligned}
\varphi^{-1}\left(\tilde{d}^{i}(\beta)\right)\left(g_{0}, g_{1}, \ldots, g_{n}\right) & =g_{0} \tilde{d}^{i}(\beta)\left(g_{0}^{-1} g_{1}, g_{1}^{-1} g_{2}, \ldots, g_{n-1}^{-1}\right) \\
& =g_{0} \beta\left(g_{0}^{-1} g_{1}, \ldots, g_{i-1}^{-1} g_{i}, \ldots, g_{n-1}^{-1} g_{n}\right) \\
& =\varphi^{-1}(\beta)\left(g_{1}, \ldots, g_{i}, \ldots, g_{n}\right) \\
& =d^{i}\left(\varphi^{-1}(\beta)\right)\left(g_{0}, g_{1}, \ldots, g_{n}\right) .
\end{aligned}
$$


For $i=n$, we have

$$
\begin{aligned}
\varphi^{-1}\left(\tilde{d}^{n}(\beta)\right)\left(g_{0}, g_{1}, \ldots, g_{n}\right) & =g_{0} \tilde{d}^{n}(\beta)\left(g_{0}^{-1} g_{1}, g_{1}^{-1} g_{2}, \ldots, g_{n-1}^{-1} g_{n}\right) \\
& =g_{0} \beta\left(g_{0}^{-1} g_{1}, \ldots, g_{n-2}^{-1} g_{n-1}\right) \\
& =\varphi^{-1}(\beta)\left(g_{0}, g_{1}, \ldots, g_{n-1}\right) \\
& =d^{n}\left(\varphi^{-1}(\beta)\right)\left(g_{0}, g_{1}, \ldots, g_{n}\right) .
\end{aligned}
$$

Together with Proposition 3.20, this proves the following comparison result.

Proposition 3.23. Let $X$ be a fibrant profinite $G$-spectrum. We equip $\operatorname{Map}\left(G^{\bullet}, X\right)$ with the cosimplicial structure of (12). There is an isomorphism of cosimplicial spectra

$$
\operatorname{Map}_{G}\left(G^{\bullet+1}, X\right) \rightarrow \operatorname{Map}\left(G^{\bullet}, X\right)
$$

which induces an isomorphism

$$
X^{h G} \cong \operatorname{Tot}\left(\operatorname{Map}\left(G^{\bullet}, X\right)\right) .
$$

There is an induced map of towers of spectra

$$
\left\{\operatorname{Tot}_{k}\left(\operatorname{Map}_{G}\left(G^{\bullet+1}, X\right)\right)\right\}_{k} \rightarrow\left\{\operatorname{Tot}_{k}(\operatorname{Map}(G \cdot X))\right\}_{k}
$$

and an isomorphism of spectral sequences from the spectral sequence of Theorem 3.17 to the spectral sequence associated to the tower of spectra

$$
\left\{\operatorname{Tot}_{k}\left(\operatorname{Map}\left(G^{\bullet}, X\right)\right)\right\}_{k} .
$$

The latter spectral sequence converges to $\pi_{t-s}\left(X^{h G}\right)$, and converges completely if $\lim _{r}^{1} E_{r}^{s, t}=0$ for all $s$ and $t$.

Remark 3.24. In order to show that the spectral sequences of the proposition are isomorphic, one could also use the following fact proved in [5] and [8]. For a profinite $G$-module $M$, let $\operatorname{Map}\left(G^{\bullet}, M\right)$ be the cochain complex given in degree $n$ by the group of continuous maps from $G^{n}$ to $M$ with differentials $\sum_{i=0}^{n}(-1)^{i} \tilde{d}^{i}$, where $\tilde{d}^{i}$ is defined as in (12) with $X$ replaced by $M$. The cohomology of this cochain complex is isomorphic to the continuous cohomology $H^{*}(G ; M)$ of $G$ with coefficients in $M$ (see [6], Proof of Theorem 3.1 on page 139). This also shows that the map $\varphi$ induces an isomorphism of spectral sequences from the $E_{2}$-terms on.

\subsection{Iterated homotopy fixed point spectra}

Let $H$ be a closed normal subgroup of $G$. The homotopy fixed points under the action of $G, H$ and $G / H$ should be related to each other. As we mentioned above, the homotopy fixed point spectrum $X^{h H}$ is in general not a profinite spectrum anymore. Hence, in general, we cannot ask for a continuous action of $G / H$ on $X^{h H}$ as a profinite spectrum. But the homotopy fixed points $X^{h G}$ do respect the continuity of the action. So for comparing $X^{h G}$ with $X^{h H}$ under its induced $G / H$-action, we assume that $K:=G / H$ is a finite group.

Starting from the model structure of simplicial $K$-sets in $[\mathbf{1 2}], \mathrm{V} \S 2$, we can define the category of $K$-spectra $\operatorname{Sp}\left(\mathcal{S}_{* K}\right)$ as in Definition 2.3. Then the method of Hovey [16] yields again a stable model structure on $\operatorname{Sp}\left(\mathcal{S}_{* K}\right)$. As above, a map $f$ in $\operatorname{Sp}\left(\mathcal{S}_{* K}\right)$ is a fibration if and only if its underlying map in $\operatorname{Sp}\left(\mathcal{S}_{*}\right)$ is a fibration of Bousfield-Friedlander spectra. 
Let $X$ be a profinite $G$-spectrum that is an $\Omega$-spectrum in $\operatorname{Sp}\left(\hat{\mathcal{S}}_{* G}\right)$. By Proposition 2.6, $X$ is also an $\Omega$-spectrum considered as a profinite $H$-spectrum. Moreover, the inclusion $H \subset G$ induces a map $E H \subset E G$ which is a trivial cofibration in $\hat{\mathcal{S}}$. Since $H$ acts freely on $E G$, we can consider $E G$ also as a cofibrant replacement of the one-point space in $\hat{\mathcal{S}}_{H}$. Hence by taking fixed points under $H$, we obtain an induced map of spectra

$$
\operatorname{Map}_{H}(E G, X)=\operatorname{Map}(E G, X)^{H} \stackrel{\sim}{\rightarrow} \operatorname{Map}(E H, X)^{H}=\operatorname{Map}_{H}(E H, X) .
$$

By mimicking the proof of Lemma 3.11 for our fixed $X$ and the trivial cofibration $E H \rightarrow E G$, we see that this map is an equivalence of $\Omega$-spectra in $\operatorname{Sp}\left(\mathcal{S}_{*}\right)$. Thus, in the following we can use the $\operatorname{spectrum} \operatorname{Map}(E G, X)^{H}$ as a model for the $H$ homotopy fixed points of $X$. By abuse of notation, we also denote this model by $X^{h H}=\operatorname{Map}(E G, X)^{H}$. The advantage of this model for the $H$-homotopy fixed points of $X$ is that it inherits a $G / H$-action from the $G$-action on $\operatorname{Map}(E G, X)$.

Furthermore, the induced map $X^{h G} \rightarrow X^{h H}$ factors through the $K$-fixed points $\left(X^{h H}\right)^{K}$, since $K$ acts trivially on $X^{h G}$. By composing with the canonical map from fixed points $\left(X^{h H}\right)^{K}=\operatorname{Map}_{K}\left(*, X^{h H}\right)$ to homotopy fixed points

$$
\left(X^{h H}\right)^{h K}=\operatorname{Map}_{K}\left(E K, X^{h H}\right),
$$

we get the map

$$
X^{h G} \rightarrow\left(X^{h H}\right)^{K} \rightarrow\left(X^{h H}\right)^{h K} .
$$

It follows almost from the definitions that this map is an equivalence. We summarize this in the following theorem in which the last assertion follows from the first and the older brother of Theorem 3.17 for finite groups.

Theorem 3.25. Let $X$ be an $\Omega$-spectrum in $\operatorname{Sp}\left(\hat{\mathcal{S}}_{* G}\right)$ and let $H$ be a normal subgroup of $G$ with finite quotient $K=G / H$. Then the map $X^{h G} \stackrel{\widetilde{\sim}}{\rightarrow}\left(X^{h H}\right)^{h K}$ is a stable equivalence in $\operatorname{Sp}\left(\mathcal{S}_{*}\right)$. Moreover, there is a spectral sequence for iterated homotopy fixed points

$$
H^{s}\left(K ; \pi_{t}\left(X^{h H}\right)\right) \Rightarrow \pi_{t-s}\left(X^{h G}\right) .
$$

\section{Morava stabilizer groups and Lubin-Tate spectra}

\section{1. $\quad E_{n}$ has a model as a profinite $G_{n}$-spectrum}

We return to our main example discussed in the introduction. For a fixed prime number $p$ and an integer $n \geqslant 1$, let $G_{n}$ denote the extended Morava stabilizer group associated to the height $n$ Honda formal group law $\Gamma_{n}$ over $\mathbb{F}_{p^{n}}$. It is a profinite group and can also be described as the group of ring spectrum automorphisms of the Lubin-Tate spectrum $E_{n}$ in the stable homotopy category. Hopkins and Miller have shown that $G_{n}$ is in fact the automorphism group of $E_{n}$ by $\mathcal{A}_{\infty}$-maps, cf. [26]. Later on, Goerss and Hopkins extended this result to the $E_{\infty}$-setting. Hence $G_{n}$ acts on the spectrum-level on $E_{n}$ by $E_{\infty}$-ring maps, cf. [11]. We will show now that there is a canonical model for $E_{n}$ in the category of profinite $G_{n}$-spectra. 
Let $B P$ be the Brown-Peterson spectrum for the fixed prime $p$. Its coefficient ring is $B P_{*}=\mathbb{Z}_{(p)}\left[v_{1}, v_{2}, \ldots\right]$, where $v_{n}$ has degree $2\left(p^{n}-1\right)$. There is a canonical map

$$
r: B P_{*} \rightarrow E_{n *}=W\left(\mathbb{F}_{p^{n}}\right)\left[\left[u_{1}, \ldots, u_{n-1}\right]\right]\left[u, u^{-1}\right]
$$

defined by $r\left(v_{i}\right)=u_{i} u^{1-p^{i}}$ for $i<n, r\left(v_{n}\right)=u^{1-p^{n}}$ and $r\left(v_{i}\right)=0$ for $i>n$. Let $I$ be an ideal in $B P_{*}$ of the form $\left(p^{i_{0}}, v_{1}^{i_{1}}, \ldots, v_{n-1}^{i_{n-1}}\right)$. Such ideals form a cofiltered system. Their images in $E_{n *}$ under $r: B P_{*} \rightarrow E_{n *}$ provide each $\pi_{t} E_{n}$ with the structure of a continuous profinite $G_{n}$-module as

$$
\pi_{t} E_{n} \cong \lim _{I} \pi_{t} E_{n} / I \pi_{t} E_{n}
$$

In fact, for $t$ odd these groups vanish, for $t$ even each quotient $\pi_{t} E_{n} / I \pi_{t} E_{n}$ is a finite discrete $G_{n}$-module and the decomposition as an inverse limit of these finite discrete $G_{n}$-modules is $G_{n}$-compatible.

For a subsystem of such ideals $I$, there are finite generalized Moore spectra $M_{I}$ with trivial $G_{n}$-action whose Brown-Peterson homology is $B P_{*}\left(M_{I}\right)=B P_{*} / I$ and which have the property $\pi_{t}\left(E_{n} \wedge M_{I}\right) \cong \pi_{t} E_{n} / I \pi_{t} E_{n}$ for all $t$ (see [14]). Moreover, there is a canonical isomorphism in the stable homotopy category

$$
E_{n} \cong \underset{I}{\operatorname{holim}} E_{n} \wedge M_{I}
$$

This observation of $[\mathbf{1 4}]$ is the starting point for all attempts to view $E_{n}$ as a continuous $G_{n}$-spectrum.

Hopkins has shown that although each $\pi_{t}\left(E_{n} \wedge M_{I}\right)$ is a finite discrete $G_{n}$-module, $G_{n}$ does not act discretely on the spectra $E_{n} \wedge M_{I}$, in the sense that there is no open normal subgroup $U$ of $G_{n}$ such that the $G_{n}$-action on the whole spectrum $E_{n} \wedge M_{I}$ factors through $G_{n} / U$ (see [4], Lemma 6.2). But a slightly less demanding statement holds. Each $E_{n} \wedge M_{I}$ and hence $E_{n}$ have a model in $\operatorname{Sp}\left(\hat{\mathcal{S}}_{* G_{n}}\right)$, i.e., models that are built out of pointed profinite $G_{n}$-spaces.

Remark 4.1. Since we are working in the world of Bousfield-Friedlander spectra, we neglect some of the rich additional structure of the spectra $E_{n}$. In particular, we form the smash products, for example $E_{n} \wedge M_{I}$, in the category of the underlying Bousfield-Friedlander spectra. A functorial construction of these smash products has for example been given in $[\mathbf{1 7}]$.

Theorem 4.2. $E_{n}$ has a model in the category of continuous profinite $G_{n}$-spectra, i.e., there is a profinite $G_{n}$-spectrum $E_{n}^{\prime}$ and a $G_{n}$-equivariant isomorphism in the stable homotopy category

$$
E_{n} \cong E_{n}^{\prime}
$$

Proof. Let $I$ be an element of the subsystem of ideals as above such that the associated finite generalized Moore spectra $M_{I}$ exists. Moreover, for each such $I$, let $\left(E_{n} \wedge M_{I}\right)_{f}$ be a functorial fibrant replacement of the underlying BousfieldFriedlander spectrum En $\wedge M_{I}$. As argued in [4], Corollary 6.4, or [14], there is an isomorphism $E_{n} \cong \operatorname{holim}_{I}\left(E_{n} \wedge M_{I}\right)_{f}$ in the stable homotopy category which is induced by the isomorphism $E_{n} \cong E_{n} \wedge \mathbb{S}^{0}$ in the stable homotopy category and the 
$G_{n}$-equivariant map

$$
E_{n} \wedge \mathbb{S}^{0} \rightarrow \underset{I}{\operatorname{holim}}\left(E_{n} \wedge M_{I}\right)_{f}
$$

whose underlying map of spectra is a stable equivalence. Now the profinite group $G_{n}$ is strongly complete (see e.g., [4], p. 330). Thus, since the homotopy groups of the $G_{n}$-spectrum $E_{n} \wedge M_{I}$ are all finite, we can apply the replacement functor of Theorem 2.7 to obtain a profinite $G_{n}$-spectrum $F_{G_{n}}^{s}\left(\left(E_{n} \wedge M_{I}\right)_{f}\right)$ built out of fibrant pointed simplicial finite $G_{n}$-sets and a $G_{n}$-equivariant map of spectra

$$
\left(E_{n} \wedge M_{I}\right)_{f} \rightarrow F_{G_{n}}^{s}\left(\left(E_{n} \wedge M_{I}\right)_{f}\right)
$$

which induces isomorphisms

$$
\pi_{t}\left(E_{n} \wedge M_{I}\right)=\pi_{t}\left(F_{G_{n}}^{s}\left(\left(E_{n} \wedge M_{I}\right)_{f}\right)\right)=\pi_{t}\left(\left|F_{G_{n}}^{s}\left(\left(E_{n} \wedge M_{I}\right)_{f}\right)\right|\right)
$$

for every $t$ and hence is a stable equivalence of spectra. We define the profinite $G_{n^{-}}$ spectrum $E_{n}^{\prime}$ to be the homotopy limit in $\operatorname{Sp}\left(\hat{\mathcal{S}}_{* G_{n}}\right)$ of the $F_{G_{n}}^{s}\left(\left(E_{n} \wedge M_{I}\right)_{f}\right)$

$$
E_{n}^{\prime}:=\underset{I}{\operatorname{holim}} F_{G_{n}}^{s}\left(\left(E_{n} \wedge M_{I}\right)_{f}\right) \in \operatorname{Sp}\left(\hat{\mathcal{S}}_{* G_{n}}\right)
$$

over the cofinal subsystem of ideals $I$ for which the $M_{I}$ exist. The profinite $G_{n^{-}}$ spectrum $E_{n}^{\prime}$ is equipped with a $G_{n}$-equivariant map of spectra

$$
E_{n} \wedge \mathbb{S}^{0} \stackrel{\simeq}{\rightarrow} \underset{I}{\operatorname{holim}}\left(E_{n} \wedge M_{I}\right)_{f} \stackrel{\simeq}{\rightarrow} \underset{I}{\operatorname{holim}} F_{G_{n}}^{s}\left(\left(E_{n} \wedge M_{I}\right)_{f}\right)=E_{n}^{\prime}
$$

which is a stable equivalence of underlying spectra. Together with the isomorphism $E_{n} \cong E_{n} \wedge \mathbb{S}^{0}$ in the stable homotopy category this proves the assertion.

Remark 4.3. The reader familiar with the work of Davis [4] will have noticed that we have even shown that each $F_{G_{n}}^{s}\left(\left(E_{n} \wedge M_{I}\right)_{f}\right)$ is a discrete $G_{n}$-spectrum. We will use this fact later in the proof of our main result.

The above theorem also implies that $E_{n}^{\prime}$ is a fibrant profinite $G$-spectrum for every closed subgroup $G$ of $G_{n}$. This allows us to define the continuous homotopy fixed point spectrum of $E_{n}$ under the action of any closed or open subgroup of $G_{n}$.

Definition 4.4. Let $G$ be a closed subgroup of $G_{n}$. The continuous homotopy fixed point spectrum $E_{n}^{h G}$ of the Lubin-Tate spectrum is the homotopy fixed point spectrum of $E_{n}^{\prime}$ considered via restriction as a profinite $G$-spectrum, i.e.,

$$
E_{n}^{h G}:=\operatorname{Map}_{G}\left(E G, E_{n}^{\prime}\right)
$$

Remark 4.5. Since $E_{n}$ is $K(n)_{*}$-local, $E_{n}^{\prime}$ is $K(n)_{*}$-local, and since taking mapping spectra preserves $K(n)_{*}$-local objects, $E_{n}^{h G}$ is a $K(n)_{*}$-local spectrum.

Before we prove Theorem 1.2 of the introduction, we collect some consequences of our construction.

Remark 4.6. We would like to point out that the idea to use continuous mapping spaces and mapping spectra in descent theory for a profinite group $H$ is not new and has been used in the context of discrete $H$-spaces, discrete $H$-spectra and towers of discrete $G$-spectra by Davis, Goerss, Jardine, Thomason and others. But since profinite spectra had not yet appeared in the picture, we provide the following results on continuous mapping spectra of $E_{n}^{\prime}$ for the convenience of the reader. 
As in the proof of Theorem 4.2, we choose and fix a subsystem of ideals such that the associated finite generalized Moore spectra $M_{I}$ are part of the canonical equivalence $E_{n} \simeq \operatorname{holim}_{I} E_{n} \wedge M_{I}$. To simplify the notation we will write

$$
E_{n, I}^{\prime}:=F_{G_{n}}^{s}\left(\left(E_{n} \wedge M_{I}\right)_{f}\right)
$$

for the fibrant profinite $G_{n}$-spectrum $F_{G_{n}}^{s}\left(\left(E_{n} \wedge M_{I}\right)_{f}\right)$ built out of simplicial finite discrete $G_{n}$-sets.

Lemma 4.7. For any closed subgroup $G$ of $G_{n}$, there is an isomorphism of $\Omega$-spectra

$$
E_{n}^{h G} \cong \operatorname{holim}_{I}\left(E_{n, I}^{\prime}\right)^{h G} .
$$

Proof. By Proposition 3.12 we have an isomorphism

$$
\operatorname{Map}_{G}\left(E G, \operatorname{holim}_{I} E_{n, I}^{\prime}\right) \cong \operatorname{holim}_{I} \operatorname{Map}_{G}\left(E G, E_{n, I}^{\prime}\right) .
$$

The first assertion now follows from the fact that we defined $E_{n}^{h G}$ to be the left hand side of this isomorphism, and that the right hand side is $\operatorname{holim}_{I}\left(E_{n, I}^{\prime}\right)^{h G}$.

Let $S$ be a profinite set and let $S=\lim _{\alpha} S_{\alpha}$ be a presentation of $S$ as a limit of finite sets. We can consider $S$ also as a constant simplicial profinite set with identities as face and degeneracy maps. Then the constructions of the previous section give us $\operatorname{spectra} \operatorname{Map}\left(S, E_{n}^{\prime}\right)$ and $\operatorname{Map}\left(S, E_{n, I}^{\prime}\right)$ for every $I$. The main case of interest for us is the one where $S=G^{j}$ is the $j$-fold product of a closed subgroup $G$ of $G_{n}$ for an integer $j \geqslant 0$.

Lemma 4.8. There is an isomorphism of $\Omega$-spectra

$$
\operatorname{Map}\left(S, E_{n}^{\prime}\right) \cong \underset{I}{\operatorname{holim}} \operatorname{Map}\left(S, E_{n, I}^{\prime}\right) .
$$

Proof. By mimicking the proof of Proposition 3.12 for $\operatorname{Map}(S,-)$, considered as a functor from $\operatorname{Sp}\left(\hat{\mathcal{S}}_{* G}\right)$ to the category of $G$-spectra, we obtain an isomorphism

$$
\operatorname{Map}\left(S, \underset{I}{\operatorname{holim}} E_{n, I}^{\prime}\right) \cong \operatorname{holim}_{I} \operatorname{Map}\left(S, E_{n, I}^{\prime}\right) .
$$

The assertion then follows from the definition of $E_{n}^{\prime}$ as $\operatorname{holim}_{I} E_{n, I}^{\prime}$.

In the following, for profinite sets $S$ and $T$, we will denote the set of continuous maps from $S$ to $T$ also by

$$
\operatorname{Map}(S, T):=\operatorname{Hom}_{\hat{\mathcal{E}}}(S, T) .
$$

Lemma 4.9. For each ideal I, the homotopy groups of the spectrum $\operatorname{Map}\left(S, E_{n, I}^{\prime}\right)$ are given by the isomorphism

$$
\pi_{*} \operatorname{Map}\left(S, E_{n, I}^{\prime}\right) \cong \operatorname{Map}\left(S, \pi_{*} E_{n, I}^{\prime}\right):=\operatorname{Hom}_{\hat{\mathcal{E}}}\left(S, \pi_{*} E_{n, I}^{\prime}\right)
$$

where the right hand side denotes the group of continuous functions from the profinite set $S$ to the finite discrete homotopy groups of $E_{n, I}^{\prime}$.

Proof. Since $E_{n, I}^{\prime}$ is an $\Omega$-spectrum that consists of simplicial finite sets, the spectrum $\operatorname{Map}\left(S, E_{n, I}^{\prime}\right)$ is isomorphic to $\operatorname{colim}_{\alpha} \operatorname{Map}\left(S_{\alpha}, E_{n, I}^{\prime}\right)$. Taking homotopy groups commutes with this colimit of fibrant spectra by [30], Lemma 5.5. Since each $S_{\alpha}$ is a finite 
set, $\operatorname{Map}\left(S_{\alpha}, E_{n, I}^{\prime}\right)$ is just a finite product of copies of $E_{n, I}^{\prime}$. This implies that taking homotopy groups also commutes with $\operatorname{Map}\left(S_{\alpha},-\right)$, i.e., there is an isomorphism

$$
\pi_{*} \operatorname{Map}\left(S_{\alpha}, E_{n, I}^{\prime}\right) \cong \operatorname{Map}\left(S_{\alpha}, \pi_{*} E_{n, I}^{\prime}\right) .
$$

Hence we obtain isomorphisms

$$
\pi_{*} \operatorname{Map}\left(S, E_{n, I}^{\prime}\right) \cong \operatorname{colim}_{\alpha} \operatorname{Map}\left(S_{\alpha}, \pi_{*} E_{n, I}^{\prime}\right) \cong \operatorname{Map}\left(S, \pi_{*} E_{n, I}^{\prime}\right) .
$$

Proposition 4.10. The homotopy groups of the spectrum $\operatorname{Map}\left(S, E_{n}^{\prime}\right)$ are given by the isomorphism

$$
\pi_{*} \operatorname{Map}\left(S, E_{n}^{\prime}\right) \cong \operatorname{Map}\left(S, \pi_{*} E_{n}^{\prime}\right):=\operatorname{Hom}_{\hat{\mathcal{E}}}\left(S, \pi_{*} E_{n}^{\prime}\right) .
$$

Proof. By Lemma 4.9, the assertion holds when we replace $E_{n}^{\prime}$ by any $E_{n, I}^{\prime}$. Moreover, $\operatorname{Map}\left(S, E_{n}^{\prime}\right)$ is isomorphic to the homotopy $\operatorname{limit}_{\operatorname{holim}} \operatorname{Map}\left(S, E_{n, I}^{\prime}\right)$ by Lemma 4.8. Hence the spectral sequence for the homotopy groups of the homotopy limit of spectra $\operatorname{Map}\left(S, E_{n}^{\prime}\right)$ has the form

$$
E_{2}^{p, q}=\lim _{I}^{p} \operatorname{Map}\left(S, \pi_{q} E_{n, I}^{\prime}\right) \Rightarrow \pi_{q-p} \operatorname{Map}\left(S, E_{n}^{\prime}\right) .
$$

Since the functor $\operatorname{Map}(S,-)$ is exact on the category of profinite abelian groups, the terms $E_{2}^{p, q}$ vanish for $p>0$ and the spectral sequence collapses. For each $q \in \mathbb{Z}$, we obtain an induced isomorphism

$$
\lim _{I} \operatorname{Map}\left(S, \pi_{q} E_{n, I}^{\prime}\right) \cong \pi_{q} \operatorname{Map}\left(S, E_{n}^{\prime}\right) .
$$

We remark that the category of profinite groups is the pro-category of finite groups. This means in particular, that the left hand side satisfies

$$
\lim _{I} \operatorname{Map}\left(S, \pi_{q} E_{n, I}^{\prime}\right) \cong \operatorname{Map}\left(S, \lim _{I} \pi_{q} E_{n, I}^{\prime}\right) .
$$

Now it remains to recall $\pi_{q} E_{n}^{\prime} \cong \lim _{I} \pi_{q} E_{n, I}^{\prime}$. This proves the assertion.

\subsection{Comparison with the construction of Devinatz and Hopkins}

Let $K(n)$ be the $n$th $p$-primary Morava $K$-theory spectrum. Its coefficient ring is given by $K(n)_{*}=\mathbb{F}_{p}\left[v_{n}, v_{n}^{-1}\right]$ where $v_{n}$ has degree $2\left(p^{n}-1\right)$. Let $\hat{L}=L_{K(n)}$ denote $K(n)_{*}$-localization in $\operatorname{Sp}\left(\mathcal{S}_{*}\right)$. As in the introduction we will adopt the notation of [1] and [4] to denote the spectra defined by Devinatz-Hopkins in [8] by $E_{n}^{d h G}$. Devinatz and Hopkins define these spectra in two steps. First they define spectra $E_{n}^{d h U}$ for open subgroups $U$ of $G_{n}$ using the fact that $G_{n} / U$ is finite and that the expected homotopy type of $\hat{L}\left(E_{n}^{d h U} \wedge E_{n}\right)$ is the one of $\operatorname{Map}\left(G_{n} / U, E_{n}\right)$. This construction depends on the specific properties of $G_{n}$ as a $p$-adic analytic profinite group, and of the important calculations of the mapping space of self-maps of $E_{n}$ by Goerss and Hopkins in [11]. (The construction of $E_{n}^{\prime}$ of course also relies on this calculation which makes it possible to define a model of $E_{n}$ on which $G_{n}$ acts by maps of spectra and not only by maps in the stable homotopy category.)

In a second step, they define spectra $E_{n}^{d h G}$ for a closed subgroup of $G_{n}$. Since $G_{n}$ is a $p$-adic analytic profinite group, it is possible to find a sequence of normal open 
subgroups of $G_{n}$

$$
G_{n}=U_{0} \nsupseteq U_{1} \supseteq \cdots \nsupseteq U_{i} \supseteq \cdots
$$

with $\bigcap_{i} U_{i}=\{e\}$. For the rest of the paper we choose a fixed sequence of such open subgroups of $G_{n}$.

Then Devinatz and Hopkins define for an arbitrary closed subgroup $G$ of $G_{n}$

$$
E_{n}^{d h G}:=\hat{L}\left(\underset{i}{\operatorname{hocolim}} E_{n}^{d h\left(U_{i} G\right)}\right)
$$

where hocolim denotes the homotopy colimit in the category of commutative $\mathbb{S}^{0}$ algebras in the category of $\mathbb{S}^{0}$-modules of $[\mathbf{9}]$.

As remarked in [8], p. 5, there is a canonical map $E_{n}^{d h G} \rightarrow E_{n}$. Since this map is $G$ equivariant and since $G$ acts trivially on $E_{n}^{d h G}$, this map factors through $E_{n}^{d h G} \rightarrow E_{n}^{G}$, i.e., through the $G$-fixed points of $E_{n}$.

Now we would like for there to be a map from the fixed points $E_{n}^{G}$ to the continuous homotopy fixed points $E_{n}^{h G}$ of Definition 4.4. But since we provided only a point-set level map $E_{n} \wedge \mathbb{S}^{0} \rightarrow E_{n}^{\prime}$, we do not have a point-set level comparison map $E_{n}^{G} \rightarrow\left(E_{n}^{\prime}\right)^{G} \rightarrow E_{n}^{h G}$. In the next section we will instead show that there is an isomorphism in the stable homotopy category between $E_{n}^{d h G}$ and $E_{n}^{h G}$ by passing through the construction of Davis in [4] and Behrens-Davis in [1]. The argument follows the suggestions of the anonymous referee whose contribution we gratefully acknowledge.

\subsection{Proof of Theorem $\mathbf{1 . 2}$}

We have defined the homotopy fixed point spectrum $E_{n}^{h G}$ for an arbitrary closed subgroup $G$ of $G_{n}$ in Definition 4.4. This proves the first assertion of (i) in Theorem 1.2. The spectral sequence of Theorem 3.17 then yields the homotopy fixed point spectral sequence

$$
H^{s}\left(G ; \pi_{t} E_{n}\right) \Rightarrow \pi_{t-s}\left(E_{n}^{h G}\right)
$$

for any closed subgroup $G$ of $G_{n}$ starting from the continuous cohomology of $G$ and converging to the homotopy groups of the homotopy fixed points of $E_{n}$ under $G$. This spectral sequence is natural in $G$. Since $G_{n}$ is a $p$-adic analytic group, so is $G$ and its continuous cohomology groups with profinite coefficients are also profinite groups, cf. [29]. Hence the $\lim ^{1}$-terms of the $E_{r}$-terms all vanish. This shows that the spectral sequence above is strongly convergent as in [8], since the spectral sequence is also conditionally convergent. This proves the first assertion of part (ii) of Theorem 1.2.

It remains to show the comparison statements of the Devinatz-Hopkins spectra $E_{n}^{d h G}$ with $E_{n}^{h G}$ and of the two spectral sequences. We will do this by showing that our homotopy fixed point spectra are equivalent to the homotopy fixed point spectra of Davis and Behrens-Davis. This shortcut is a suggestion by the anonymous referee and we gratefully acknowledge his contribution and generosity to share the outline of the following argument with us.

We denote the homotopy fixed point spectrum of Davis by $E_{n}^{h^{\prime} G}$ and refer the reader to $[\mathbf{4}]$ and $[\mathbf{1}]$ for the details of the construction and details about the properties we use in the proof. By Proposition 3.20 and Proposition 3.23, we have an isomorphism of spectra

$$
E_{n}^{h G}=\operatorname{Map}_{G}\left(E G, E_{n}^{\prime}\right) \cong \operatorname{Tot}\left(\operatorname{Map}_{G}\left(G^{\bullet+1}, E_{n}^{\prime}\right)\right) \cong \operatorname{TotMap}\left(G^{\bullet}, E_{n}^{\prime}\right) .
$$

Moreover, since $\operatorname{Map}\left(G^{\bullet}, E_{n}^{\prime}\right) \cong \operatorname{Map}_{G}\left(G^{\bullet+1}, E_{n}^{\prime}\right)$ is a fibrant cosimplicial fibrant 
spectrum, the canonical map

$$
\operatorname{Tot} \operatorname{Map}\left(G^{\bullet}, E_{n}^{\prime}\right) \rightarrow \underset{\Delta}{\operatorname{holim}} \operatorname{Map}\left(G^{\bullet}, E_{n}^{\prime}\right)
$$

is an equivalence of spectra by [30], Lemma 5.25. By our construction of $E_{n}^{\prime}$ as the homotopy limit of the $E_{n, I}^{\prime}$, we obtain a further equivalence of spectra

$$
E_{n}^{h G} \simeq \underset{I}{\operatorname{holim}} \underset{\Delta}{\operatorname{holim}} \operatorname{Map}\left(G^{\bullet}, E_{n, I}^{\prime}\right) .
$$

The collection of ideals $I$ contains a descending chain of ideals $\left\{I_{0} \supset I_{1} \supset \ldots\right\}$ with an associated tower of generalized Moore spectra $\left\{M_{I_{0}} \leftarrow M_{I_{1}} \leftarrow \cdots\right\}$ which is cofinal in the sense that there is still an isomorphism of spectra in the stable homotopy category $E_{n} \cong \operatorname{holim}_{I_{k}} E_{n} \wedge M_{I_{k}}$. Choosing this tower of generalized Moore spectra $\left\{M_{I_{k}}\right\}$ makes it possible to compare our construction with the one of [4].

By its construction each $E_{n, I_{k}}^{\prime}:=F_{G_{n}}^{s}\left(\left(E_{n} \wedge M_{I_{k}}\right)_{f}\right)$ is also a discrete $G$-spectrum in the sense of $[4]$, since it is built out of pointed simplicial finite discrete $G$-sets. Hence, for $j \geqslant 0$, we can write

$$
\begin{aligned}
\operatorname{Map}\left(G^{j}, E_{n, I_{k}}^{\prime}\right) & \cong \operatorname{colim}_{i} \operatorname{Map}\left(\left(G /\left(G \cap U_{i}\right)\right)^{j}, E_{n, I_{k}}^{\prime}\right) \\
& \cong \operatorname{colim}_{i} \prod_{\left(G /\left(G \cap U_{i}\right)\right)^{j}} E_{n, I_{k}}^{\prime} \\
& \cong \operatorname{Map}_{c}\left(G^{j}, E_{n, I_{k}}^{\prime}\right)
\end{aligned}
$$

where the last expression $\operatorname{Map}_{c}\left(G^{j}, E_{n, I_{k}}^{\prime}\right)$ denotes the continuous mapping spectrum of [4] for the discrete $G$-spectrum $E_{n, I_{k}}^{\prime}$. The collection $\left\{\operatorname{Map}_{c}\left(G^{j}, E_{n, I_{k}}^{\prime}\right)\right\}_{j \geqslant 1}$ forms a cosimplicial spectrum defined in [4] as follows. Let $\Gamma_{G}(-)$ be the functor on discrete $G$ spectra $X \mapsto \operatorname{Map}_{c}(G, U(X))$, where $U$ denotes the functor that forgets the $G$-action. The spectrum $\operatorname{Map}_{c}(G, X)$ is equipped with a $G$-action given by $(g f)(h)=f(h g)$. This action turns $\operatorname{Map}_{c}(G, X)$ itself into a discrete $G$-spectrum. Iterated application of $\Gamma_{G}(-)$ defines a cosimplicial object $\left(\Gamma_{G} X\right)^{\bullet}$ of discrete $G$-spectra with

$$
\left(\Gamma_{G} X\right)^{j} \cong \operatorname{Map}_{c}\left(G^{j+1}, X\right)
$$

Now one can check as for discrete $G$-modules that the $G$-fixed points of $\left(\Gamma_{G} X\right)^{\bullet}$ are given by the cosimplicial spectrum $\operatorname{Map}_{c}\left(G^{\bullet}, X\right)$ given in degree $j$ by $\operatorname{Map}_{c}\left(G^{j}, X\right)$ and with coface maps given by

$$
\bar{d}^{i}(\beta)\left(g_{1}, \ldots, g_{j+1}\right)= \begin{cases}g_{1} \beta\left(g_{2}, \ldots, g_{j+1}\right) & : i=0 \\ \beta\left(g_{1}, \ldots, g_{i} g_{i+1}, \ldots, g_{j+1}\right) & : 1 \leqslant i \leqslant j \\ \beta\left(g_{1}, \ldots, g_{j}\right) & : i=j+1\end{cases}
$$

Hence by Proposition 3.23 and isomorphism (14), we obtain an isomorphism of cosimplicial spectra

$$
\operatorname{Map}_{G}\left(G^{\bullet+1}, E_{n, I_{k}}^{\prime}\right) \cong \operatorname{Map}\left(G^{\bullet}, E_{n, I_{k}}^{\prime}\right) \cong \operatorname{Map}_{c}\left(G^{\bullet}, E_{n, I_{k}}^{\prime}\right) \cong \operatorname{Map}_{c}\left(G^{\bullet+1}, E_{n, I_{k}}^{\prime}\right)^{G}
$$

This implies that we have an equivalence

$$
E_{n}^{h G} \simeq \underset{k}{\operatorname{holim}} \underset{\Delta}{\operatorname{holim}} \operatorname{Map}\left(G^{\bullet}, E_{n, I_{k}}^{\prime}\right) \cong \underset{k}{\operatorname{holim}} \underset{\Delta}{\operatorname{holim}} \operatorname{Map}_{c}\left(G^{\bullet+1}, E_{n, I_{k}}^{\prime}\right)^{G} .
$$

By [4], since each $E_{n, I_{k}}^{\prime}$ is by construction a fibrant spectrum, we obtain an equivalence

$$
\underset{k}{\operatorname{holim}} \operatorname{holim}_{\Delta} \operatorname{Map}_{c}\left(G^{\bullet+1}, E_{n, I_{k}}^{\prime}\right)^{G} \simeq E_{n}^{h^{\prime} G}
$$


This shows that there is an equivalence of spectra $E_{n}^{h G} \simeq E_{n}^{h^{\prime} G}$. Hence together with the equivalence $E_{n}^{h^{\prime} G} \simeq E_{n}^{d h G}$ of Behrens-Davis in [1] , Theorem 8.2.1, we get a sequence of equivalences of spectra

$$
E_{n}^{h G} \simeq E_{n}^{h^{\prime} G} \simeq E_{n}^{d h G}
$$

Finally, we have to compare the spectral sequences. Isomorphism (16) implies that there is an isomorphism of towers of cosimplicial spectra

$$
\left\{\operatorname{Map}\left(G^{\bullet}, E_{n, I_{k}}^{\prime}\right)\right\}_{k} \cong\left\{\operatorname{Map}_{c}\left(G^{\bullet+1}, E_{n, I_{k}}^{\prime}\right)^{G}\right\}_{k} .
$$

The homotopy spectral sequence converging to

$$
\pi_{*}\left(\operatorname{holim}_{\Delta} \operatorname{holim}_{k} \operatorname{Map}_{c}\left(G^{\bullet+1}, E_{n, I_{k}}^{\prime}\right)^{G}\right)
$$

corresponding to the homotopy limit over $\Delta$ of holim $k$ of the right hand side is isomorphic from the $E_{2}$-terms on to the homotopy fixed point spectral sequence converging to $\pi_{*}\left(E_{n}^{h^{\prime} G}\right)$ of [4]. By Proposition 3.20 and Proposition 3.23 and by the choice of the sequence of ideals $I_{k}$, the homotopy spectral sequence converging to $\pi_{*}\left(\operatorname{holim}_{\Delta} \operatorname{holim}_{k} \operatorname{Map}\left(G^{\bullet}, E_{n, I_{k}}^{\prime}\right)_{k}\right)$ corresponding to the homotopy limit over $\Delta$ of $\operatorname{holim}_{k}$ of the left hand side is isomorphic from the $E_{2}$-terms on to the homotopy fixed point spectral sequence converging to $\pi_{*}\left(E_{n}^{h G}\right)$. Hence isomorphism (18) implies that the homotopy fixed point spectral sequence converging to $\pi_{*}\left(E_{n}^{h G}\right)$ is isomorphic from the $E_{2}$-terms on to the homotopy fixed point spectral sequence converging to $\pi_{*}\left(E_{n}^{h^{\prime} G}\right)$. By the spectral sequence comparison result of Behrens-Davis in [1], Theorem 8.2.5, the latter spectral sequence is isomorphic from the $E_{2}$-terms on to the $K(n)_{*}$-local $E_{n}$-Adams spectral sequence of [8] converging to $\pi_{*}\left(E_{n}^{d h G}\right)$. Hence overall we obtain an isomorphism of spectral sequences from the $E_{2}$-terms on of the $K(n)_{*}$-local $E_{n}$-Adams spectral sequence of $[\mathbf{8}]$ and the homotopy fixed point spectral sequence converging to $\pi_{*}\left(E_{n}^{h G}\right)$. This finishes the proof of Theorem 1.2.

Corollary 4.11. Let $G$ be a closed subgroup of $G_{n}$, and $K$ be a closed normal subgroup of $G$ such that $G / K$ is finite. Then $E_{n}^{h G}$ is naturally equivalent to $\left(E_{n}^{h K}\right)^{h G / K}$. There is a strongly convergent spectral sequence for iterated homotopy fixed points

$$
H^{*}\left(G / K ; \pi_{*} E_{n}^{h K}\right) \Rightarrow \pi_{*} E_{n}^{h G} .
$$

Proof. The equivalence between $E_{n}^{h G}$ and $\left(E_{n}^{h K}\right)^{h G / K}$ and the existence of the spectral sequence follow from Theorem 3.25 applied to $E_{n}^{\prime}$ of Theorem 4.2. The strong convergence follows from the conditional convergence of Theorem 3.25 and the facts that $G / K$ is compact $p$-adic analytic, that each $\pi_{k}\left(E_{n}^{d h K}\right)$ is a finite group by [6], Lemma 3.5 , and from the isomorphism $\pi_{*}\left(E_{n}^{h K}\right) \cong \pi_{*}\left(E_{n}^{d h K}\right)$ given by Theorem 1.2.

\section{References}

[1] M. Behrens, D. G. Davis, The homotopy fixed point spectra of profinite Galois extensions, Trans. Amer. Math. Soc. 362 (2010), 4983-5042.

[2] A. K. Bousfield, E. M. Friedlander, Homotopy of $\Gamma$-spaces, spectra and bisimplicial sets, in: Geometric Applications of Homotopy Theory (Evanston, IL, 1977), vol. II, Lecture Notes in Mathematics 658, Springer, Berlin, 1978, 80130. 
[3] A. K. Bousfield, D. M. Kan, Homotopy limits, Completions and Localizations, Lecture Notes in Mathematics 304, Springer-Verlag, Berlin-New York, 1972.

[4] D. G. Davis, Homotopy fixed points for $L_{K(n)}\left(E_{n} \wedge X\right)$ using the continuous action, J. Pure Appl. Algebra 206 (2006), 322-354.

[5] E. S. Devinatz, Morava's change of rings theorem, in: The Čech centennial (Boston, MA, 1993), Contemp. Math. 181, Amer. Math. Soc., Providence, 1995, 83-118.

[6] E. S. Devinatz, A Lyndon-Hochschild-Serre spectral sequence for certain homotopy fixed point spectra, Trans. Amer. Math. Soc. 357 (2005), 129-150.

[7] E. S. Devinatz, M. J. Hopkins, The action of the Morava stabilizer group on the Lubin-Tate moduli space of lifts, Amer. J. Math. 117 (1995), 669-710.

[8] E. S. Devinatz, M. J. Hopkins, Homotopy fixed point spectra for closed subgroups of the Morava stabilizer groups, Topology 43 (2004), 1-47.

[9] A. D. Elmendorf, I. Kriz, M. A. Mandell, J. P. May, Rings, Modules and Algebras in Stable Homotopy Theory, Amer. Math. Soc. Surveys and Monographs 47, Amer. Math. Soc., Providence, 1997.

[10] H. Fausk, Equivariant homotopy theory for pro-spectra, Geom. Topol. 12 (2008), 103-176.

[11] P. G. Goerss, M. J. Hopkins, Moduli spaces of commutative ring spectra, in: Structured ring spectra, 151-200, London Math. Soc. Lecture Note Ser. 315, Cambridge Univ. Press, Cambridge, 2004.

[12] P. G. Goerss, J. F. Jardine, Simplicial Homotopy Theory, Birkhäuser Verlag, Basel, 1999.

[13] P. S. Hirschhorn, Model Categories and Their Localizations, Mathematical Surveys and Monographs 99, Amer. Math. Soc., Providence, 2003.

[14] M. J. Hopkins, M. Mahowald, H. Sadofsky, Constructions of elements in Picard groups, in: Topology and Representation Theory (Evanston, IL, 1992), Amer. Math. Soc., Providence, 1994, 89-126.

[15] M. Hovey, Model Categories, Mathematical Surveys and Monographs 63, Amer. Math. Soc., Providence, 1999.

[16] M. Hovey, Spectra and symmetric spectra in general model categories, J. Pure Appl. Algebra 165 (2001), 63-127.

[17] J. F. Jardine, Generalized Etale Cohomology Theories, Progress in Mathematics 146, Birkhäuser Verlag, Basel, 1997.

[18] J. Lubin, J. Tate, Formal moduli for one-parameter formal Lie groups, Bull. Soc. Math. France 94 (1966), 49-59.

[19] H. R. Miller, On relations between Adams spectral sequences, with an application to the stable homotopy of a Moore space, J. Pure Appl. Algebra 20 (1981), 287-312.

[20] J. P. May, Simplicial objects in algebraic topology, Reprint of the 1967 original. Chicago Lectures in Mathematics. University of Chicago Press, Chicago, 1992. 
[21] J. Morava, Noetherian localisations of categories of cobordism comodules, Ann. of Math. 121 (1985), 1-39.

[22] N. Nikolov, D. Segal, On finitely generated profinite groups. I. Strong completeness and uniform bounds, Ann. of Math. 165 (2007), 171-238.

[23] G. Quick, Stable étale realization and étale cobordism, Adv. Math. 214 (2007), 730-760.

[24] G. Quick, Profinite G-spectra, Homology Homotopy Appl. 15(1) (2013), 151189.

[25] D. G. Quillen, Homotopical algebra, Lecture Notes in Mathematics 43, Springer-Verlag, Berlin-New York, 1967.

[26] C. Rezk, Notes on the Hopkins-Miller theorem, in: M. Mahowald, S. Priddy (Eds.), Homotopy Theory via Algebraic Geometry and Group Representations (Evanston, IL, 1997), Contemp. Math. 220, Amer. Math. Soc., Providence, 1998, 313-366.

[27] L. Ribes, P. Zalesskii, Profinite Groups, Ergebnisse der Mathematik und ihrer Grenzgebiete 40, Springer-Verlag, Berlin, 2000.

[28] J. P. Serre, Cohomologie Galoisienne, Lecture Notes in Mathematics 5, Springer-Verlag, Berlin-New York, 1965.

[29] P. Symonds, T. Weigel, Cohomology of $p$-adic analytic groups, in: M.du Sautoy, et al., (Eds.), New Horizons in pro-p Groups, Progress in Mathematics 184, Birkhäuser Boston, Boston, 2000, 349-410.

[30] R. W. Thomason, Algebraic K-Theory and Etale Cohomology, Ann. Sci. E.N.S. 18 (1985), 437-552.

Gereon Quick gquick@math.harvard.edu

Department of Mathematics, Harvard University, One Oxford Street, Cambridge, MA 02138, USA 